\title{
THE FOUNDATIONS OF A THEORY IN THE CALCULUS OF VARIATIONS IN THE LARGE*
}

\author{
BY \\ MARSTON MORSE
}

\section{INTRODUCTION}

The conventional object in a paper on the calculus of variations is the investigation of the conditions under which a maximum or minimum of a given integral occurs. Writers have accordingly done little with extremal segments that have contained more than one point conjugate to a given point. An extended theory is needed for several reasons.

One reason is that in applying the calculus of variations to geodesics, or to that very general class of dynamical systems or differential equations which may be put in the form of the Euler equations, it is by no means a minimum or a maximum that is always sought. For example, if in the problem of two bodies we make use of the corresponding Jacobi principle of least action $\dagger$ the ellipses which thereby appear as extremals always have pairs of conjugate points on them, and do not accordingly give a minimum to the integral relative to neighboring closed curves, so that no example of periodic motion would be found by a search for a minimum of the Jacobi integral. In general if one is looking for extremals joining two points or periodic extremals deformable into a given closed curve, the a priori expectation, as justified by the results of this paper, in general problems, would seem to be that more solutions would not give an extremum than would give an extremum.

A second reason for the study of extremal segments and periodic extremals that do not furnish an extremum for the integral is that if the ultimate object sought is an extremum, the existence of such extrema is tied up "in the large" with the existence of extremals which do not furnish extrema. It is one of the purposes of this paper to show the relations in the large between all sorts of extremals joining two fixed points, or deformable into a given closed curve.

A first type of a priori existence theorem is Hilbert's theorem concerning

\footnotetext{
* Presented to the Society in part December 30,1924, under the title Relations in the large between the numbers of extremals of different types joining two fixed points, and in part December 29, 1926, under the title The type number and rank of a closed extremal, and the consequent theory in the large; received by the editors January $15,1927$.

† See P. Appell, Traite de Mécanique Rationnelle, Paris, 1919, vol. 1, pp. 547-548.
} 
the absolute minimum. ${ }^{*}$ Reference should be given to the more recent work of Tonelli, $\nmid$ also on the absolute minimum. Other references to studies of this sort will be found in the standard treatises. Birkhoff $\ddagger$ effectively departed from the study of the absolute minimum alone, when he stated his "minimax principle," and applied it to find closed trajectories that do not give a minimum to his "least action" integral. The present writer\& has shown that Birkhoff's points of minimum and minimax appear as two types of critical points among $n+1$ such types, and has replaced Birkhoff's inequality relation by $n$ inequality relations and one equality relation. One of the objects of the present paper is to show how these $n+1$ relations between critical points can be translated into relations between different types of extremals.

It was necessary to develop for the first time a complete parallelism between types of critical points and types of extremals. It was found that the type of an extremal segment whose ends were not conjugate was completely determined by the number of mutual conjugate points on the segment. In the case where the end points were conjugate it was necessary to bring in the envelope theory.

Turning to periodic extremals it was found, even in the most general cases, that conjugate points would not serve to determine the type of a periodic extremal $g$, but that other relations of $g$ to neighboring extremals had to be brought in. A periodic extremal was called degenerate if the corresponding Jacobi differential equation possessed periodic solutions not identically zero. It is shown for the first time how a parameter may be introduced into the integrand in such a fashion that the degenerate periodic extremal disappears and non-degenerate periodic extremals, or no periodic extremals, take its place.

This theory is brought to a head in two applications, one "in the large," giving relations between different types of extremals, and one "in the small," showing how the type of a given extremal may be characterized in a third way, in terms of the possibility or impossibility of deformations of $m$ parameter families of neighboring extremals into families of lower dimensionality. This deformation theory was made possible by the application

\footnotetext{
* Bolza, Vorlesungen über Variationsrechnung, 1909, pp. 419-437. Further references to Bolza will be indicated by the letter $B$.

$\dagger$ Tonelli, Fondamenti di Calcolo delle Variazioni, vol. 2.

$\ddagger$ Birkhoff, Dynamical syslems with two degrees of freedom, these Transactions, vol. 18 (1917), p. 240.

8 Marston Morse, Relations between the critical points of a real function of $n$ independent variables, these Transactions, vol. 27 (1925), pp. 345-396.
} 
of certain powerful theorems of analysis situs. It is believed that this deformation theory will serve as the basis for an even more extended theory "in the large."

Part I. The type number of an extremal segment

1. The integrand $F(x, y, \dot{x}, \dot{y})$ and the function $J\left(v_{1}, \cdots, v_{n}\right)$. Let $(x, y)$ be any point in an open two-dimensional region $S$ of the $x, y$ plane. Let there be given a function $F(x, y, \dot{x}, \dot{y})$ of class $C^{\prime \prime \prime}$ (B, loc. cit., p. 193), and positively homogeneous in $\dot{x}$ and $\dot{y}$ of dimension one, for $(x, y)$ in $S$ and $\dot{x}$ and $\dot{y}$ any two numbers not both zero. Corresponding to the calculus of variations problem in the parametric form with integral $J$, and with $F(x, y, \dot{x}, \dot{y})$ as the integrand, let there be given an extremal $g$ of class $C^{\prime \prime \prime}$ (B, p. 191), without multiple points, passing from a point $A$ to a point $B$, and such that along $g$ we have (B, p. 196)

$$
F_{1}(x, y, \dot{x}, \dot{y})>0 .
$$

Let the arc length along $g$, measured from $A$ toward $B$, be denoted by $u$. Let

$$
u_{1}, u_{2}, \cdots, u_{n}
$$

be $n$ values of $u$ increasing with their subscripts, and corresponding to points on $g$ between $A$ and $B$. Denote the value of $u$ at $A$ by $u_{0}$, and its value at $B$ by $u_{n+1}$. We suppose the points (2) so chosen on $g$ that no one of the closed segments of $g$ bounded by successive points of the set

$$
u_{0}, u_{1}, \cdots, u_{n+1}
$$

contains a conjugate point of either end poirt of that segment. Let there be given $n$ short arcs of class $C^{\prime \prime \prime}$, say $h_{1}, h_{2}, \cdots, h_{n}$, passing respectively through the points of $g$ at which $u$ takes on the values (2), arcs not tangent to $g$ at these points. Let positive senses be assigned to $h_{1}, h_{2}, \cdots, h_{n}$ in such a fashion that the positive tangent to $g$ at $u=u_{i}$ has to be turned through a positive angle less than $\pi$ to coincide with the positive tangent to $h_{i}$ at the same point. Let $v_{i}$ be the arc length measured along $h_{i}$ in $h_{i}$ 's positive sense from the point $u=u_{i}$ on $g$. We regard $\left(u_{i}, v_{i}\right)$ as representing the point on $h_{i}$ at the distance $v_{i}$ from $g$.

If each $v_{i}$ be sufficiently small in absolute value the points

$$
A,\left(u_{1}, v_{1}\right),\left(u_{2}, v_{2}\right), \cdots,\left(u_{n}, v_{n}\right), B
$$

can be successively joined by unique extremal segments neighboring $g$. 
Let the integral $J$ taken along this broken extremal joining $A$ to $B$ be denoted by

$$
J\left(v_{1}, \cdots, v_{n}\right) .
$$

Corresponding to the extremal $g$ the function (5) will be shown to have a critical point for $\left(v_{1}, \cdots, v_{n}\right)=(0,0, \cdots, 0)$, that is a point at which all of its partial derivatives are zero. We will also investigate the terms of second order in the expansion of the function $(5)$ about $(0,0, \cdots, 0)$.

2. A transformation of the problem. We introduce the following lemma which simplifies the problem.

Lemma. It is possible to make a transformation $T$ from the $(x, y)$ plane to the $(u, v)$ plane with the following properties:

(A) Under $T$ the extremal $g$ is carried into a portion $\gamma$ of the $u$ axis bounded by $u=u_{0}$ and $u=u_{n+1}$.

(B) The transformation establishes a one-to-one correspondence between a suitably chosen region of the $(x, y)$ plane enclosing $g$, and a region $R_{1}$ of the $(u, v)$ plane enclosing $\gamma$.

(C) The transformation carries the arcs $h_{1}, h_{2}, \cdots, h_{n}$ into straight line segments on which $u=u_{1}, u_{2}, \cdots, u_{n}$, respectively.

(D) The transformation is representable in the form

$$
x=x(u, v), y=y(u, v)
$$

where $x(u, v), y(u, v)$ are of class $C^{\prime \prime \prime}$ in $R_{1}$ and possess there a positive jacobian.

(E) The transformation preserves arc lengths along $g$ and $h_{1}, h_{2}, \cdots, h_{n}$.

In the first preparation of this paper the complete details of the proof of this lemma were given, but second thought makes it seem not too much to leave to the reader.

3. The integral in the $(u, v)$ plane. Under the transformation of the preceding lemma the integrand $F(x, y, \dot{x}, \dot{y})$ can be replaced by a new integrand $G(u, v, \dot{u}, \dot{v})$, where $\dot{x}, \dot{y}, \dot{u}, \dot{v}$ stand for derivatives of $x, y, u, v$, respectively, with respect to a parameter $t$ (B, p. 344). We are concerned with properties of the extremal segment $\gamma$, namely,

$$
v \equiv 0, u_{0} \leqq u \leqq u_{n+1},
$$

which corresponds under the preceding transformation $T$ to the extremal $g$ in the $(x, y)$ plane. For the present we need only consider curves on which $\dot{u}>0$, and set

$$
G\left(u, v, 1, v^{\prime}\right)=f\left(u, v, v^{\prime}\right), \quad v^{\prime}=\frac{d v}{d u}
$$


thus defining $f\left(u, v, v^{\prime}\right)$ for all points $(u, v)$ neighboring $\gamma$, and all numbers $v^{\prime}$. For this domain $f\left(u, v, v^{\prime}\right)$ is of class $C^{\prime \prime \prime}$. The points which are enumerated in $\$ 1,(4)$, here correspond to points whose actual coördinates are

$$
\left(u_{0}, 0\right),\left(u_{1}, v_{1}\right), \cdots,\left(u_{n}, v_{n}\right),\left(u_{n+1}, 0\right) .
$$

Subject to the limitation that we deal here only with curves on which $\dot{u}>\mathbf{0}$, the integral $J$ of $\$ 1$ becomes here

$$
J=\int_{u_{0}}^{u_{n+1}} f\left(u, v, v^{\prime}\right) d u
$$

and the function

$$
J\left(v_{1}, \cdots, v_{n}\right)
$$

is the value of the integral (4) taken along the successive extremals joining the successive points of (3), varying the coördinates $v_{i}$, but holding all coördinates $u_{i}$ fast. It should be expressly noted that the function (5) is not a new function set up for the first time in the $(u, v)$ plane, but that it is identical with the function $J\left(v_{1}, \cdots, v_{n}\right)$ defined in $\$ 1$.

In terms of $f\left(u, v, v^{\prime}\right)$, and for the extremal $\gamma$ given by (1), we define the functions $P(u), Q(u)$, and $R(u)$ in the usual way (B, p. 55). These functions are of class $C^{\prime}$ along $\gamma$. Because of the assumption that $F_{1}>0$ along $g$, it follows that

$$
R(u)>0
$$

along $\gamma$. The Jacobi differential equation corresponding to the extremal segment $\gamma$ will be written in the well known form

$$
R w^{\prime \prime}+R^{\prime} w^{\prime}+\left(Q^{\prime}-P\right) w=0,
$$

with $w$ the dependent variable, and $u$ the independent variable. We are always going to write J. D. E. for (7).

4. The second partial derivatives of $J\left(v_{1}, \cdots, v_{n}\right)$. We shall see presently that $J\left(v_{1}, \cdots, v_{n}\right)$ has a critical point when $\left(v_{1}, \cdots, v_{n}\right)=(0, \cdots, 0)$. To determine the nature of this critical point we proceed to the determination of the second partial derivatives of $J\left(v_{1}, \cdots, v_{n}\right)$. To that end we represent the family of extremals which join the points

$$
\left(u_{i}, v_{i}\right),\left(u_{i+1}, v_{i+1}\right) \quad(i=0,1, \cdots, n)
$$

in the form

$$
v=r^{i}\left(u, v_{i}, v_{i+1}\right), u_{i} \leqq u \leqq u_{i+1},
$$


where it is understood that the coördinates $u_{i}$ and $u_{i-1}$ are held fast. The functions

$$
r^{i}, r_{u}^{i}, r_{u u}^{i}
$$

will be of class $C^{\prime}$ in all of their arguments for $u$ on the interval in (2), and for $v_{i}$ and $v_{i+1}$ neighboring zero (B, p. 73 and p. 307).

We shall understand by $w_{\mu \nu}(u)$, a solution of the J. D. E., such that

(4) $w_{\mu \nu}\left(u_{\mu}\right)=0, w_{\mu \nu}\left(u_{\nu}\right)=1(|\mu-\nu|=1, \mu, \nu=0,1,2, \cdots, n+1)$.

Because of the fact that the functions (2) satisfy the identities

it follows that

$$
\begin{aligned}
v_{i} & \equiv r^{i}\left(u_{i}, v_{i}, v_{i+1}\right), \\
v_{i+1} & \equiv r^{i}\left(u_{i+1}, v_{i}, v_{i+1}\right),
\end{aligned}
$$

$$
w_{i+1, i}(u) \equiv r_{v i}^{i}(u, 0,0),
$$

$$
w_{i, i+1}(u) \equiv r_{v_{i+1}}^{i}(u, 0,0)
$$

Differentiation of the integral $J$, and integration by parts in the usual way will now give

(7) $J_{v_{i}}\left(v_{1}, \cdots, v_{n}\right)=f_{v^{\prime}}\left[u_{i}, v_{i}, r_{u}^{i-1}\left(u_{i}, v_{i-1}, v_{i}\right)\right]-f_{v^{\prime}}\left[u_{i}, v_{i}, r_{u}^{i}\left(u_{i}, v_{i}, v_{i+1}\right)\right]$.

Here it is understood that

$$
i=1,2, \cdots, n, v_{0}=0, v_{n+1}=0 .
$$

From (7) we see that all the partial derivatives of $J\left(v_{1}, \cdots, v_{n}\right)$ are zero at $(0, \cdots, 0)$, and note that $J\left(v_{1}, \cdots, v_{n}\right)$ is of class $C^{\prime}$ in the neighborhood of $(0, \cdots, 0)$.

From (7) we see that

$$
J_{v_{i}, v_{j}}=0,|i-j| \neq 1,0 .
$$

The remaining second partial derivatives will be evaluated at $(0, \cdots, 0)$. Evaluation at the latter point will be indicated by a subscript zero preceding the partial derivative. We obtain the following results:*

$$
\begin{aligned}
{ }_{0} J_{v_{i}, v_{i}} & =R\left(u_{i}\right)\left[r_{u, v_{i}}^{i-1}\left(u_{i}, 0,0\right)-r_{u, v_{i}}^{i}\left(u_{i}, 0,0\right)\right] \\
& =R\left(u_{i}\right)\left[w_{i-1, i}^{\prime}\left(u_{i}\right)-w_{i+1, i}^{\prime}\left(u_{i}\right)\right] \quad(i=1,2, \cdots, n) ;
\end{aligned}
$$

* A. Dresden has given complete formulas for the second partial derivatives of the extremal integral (Bolza, p. 310). Use has not been made of this work, however, because the formulas given in the present paper need not have the general form given by Dresden, and do need a different notation. 


$$
\begin{array}{ll}
a J_{v_{i}, v_{i+1}}=-R\left(u_{i}\right) w_{i, i+1}^{\prime}\left(u_{i}\right) & (i=1,2, \cdots, n-1) ; \\
a J_{v_{i+1} v_{i}}=R\left(u_{i+1}\right) w_{i+1, i}^{\prime}\left(u_{i+1}\right) & (i=1,2, \cdots, n-1) .
\end{array}
$$

The last two partial derivatives are necessarily equal, as can be proved directly from the properties of the J. D. E.

The matrix whose elements are

$$
a_{i, j}=a J_{v_{i}, v_{j}} \quad(i, j=1,2, \cdots, n)
$$

has now been determined. Note first that all elements in the $i$ th row of this matrix have the factor $R\left(u_{i}\right)$. Let this factor be removed from the $i$ th row $(i=1,2, \cdots, n)$. We will write down the resulting matrix for a typical case, $n=5$.

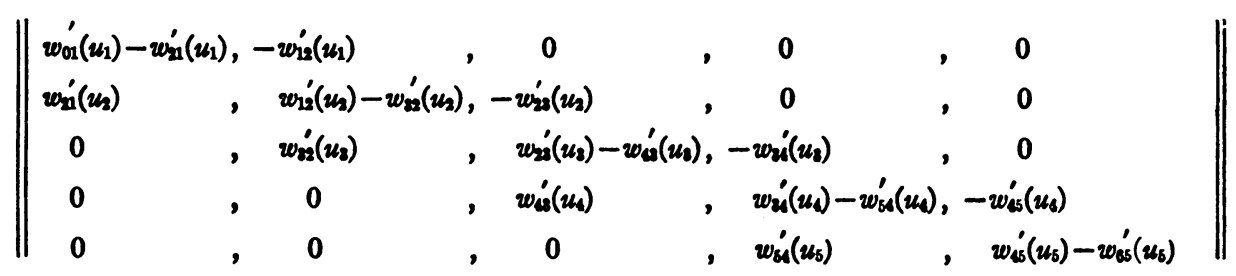

5. The rank of the extremal segment $g$. We prove the following theorem:

TheOREM 1. Let the function $J\left(v_{1}, \cdots, v_{n}\right)$ be set up for the extremal segment $g$, as described in $\$ 1$. The matrix a, whose elements are

$$
a_{i j}=a_{v_{i} v_{j}},
$$

is of rank $n$ if the final point of $g$ is not among the conjugate points of the initial point of $g$. Otherwise a is of rank $n-1$.

To prove this theorem let us turn to the $(u, w)$ plane of the J. D. E., and in that plane join the successive points

$$
\left(u_{0}, 0\right)\left(u_{1}, c_{1}\right), \cdots,\left(u_{n}, c_{n}\right)\left(u_{n+1}, 0\right),
$$

by curve segments representing solutions of the J. D. E. The successive segments are of the form

$$
w=c_{i} w_{i+1, i}(u)+c_{i+1} w_{i, i+1}(u), \quad u_{i} \leqq u \leqq u_{i+1},
$$

where

$$
i=0,1, \cdots, n, \quad c_{0}=0, \quad c_{n+1}=0 .
$$

Let the curve obtained by combining the successive segments (1) be denoted by $\lambda$. A necessary and sufficient condition that the point $\left(u_{0}, 0\right)$ be conjugate to $\left(u_{n+1}, 0\right)$ is that among the curves $\lambda$ there exist at least one, not 
$w \equiv 0$, that has no corners at the junctions of its successive segments. The $n$ conditions that $\lambda$ have no corners at the junctions of these segments are

$$
\left[c_{i-1} w_{i, i-1}^{\prime}\left(u_{i}\right)+c_{i} w_{i-1, i}^{\prime}\left(u_{i}\right)\right]-\left[c_{i} w_{i+1, i}^{\prime}\left(u_{i}\right)+c_{i+1} w_{i, i+1}^{\prime}\left(u_{i}\right)=0\right],
$$

where

$$
i=1,2, \cdots, n, \quad c_{0}=c_{n+1}=0 .
$$

Equations (3) may be written in the form

$$
c_{i-1} w_{i, i-1}^{\prime}\left(u_{i}\right)+c_{i}\left[w_{i-1, i}^{\prime}\left(u_{i}\right)-w_{i+1, i}^{\prime}\left(u_{i}\right)\right]-c_{i+1} w_{i, i+1}^{\prime}\left(u_{i}\right)=0,
$$

subject again to the conditions (4).

Let the matrix of the coefficients of $\left(c_{1}, \cdots, c_{n}\right)$ in (5) be denoted by $w$ and the value of the corresponding determinant by $|w|$. With the aid of (9), (10), (11), and (12) of $\$ 4$ we obtain the following equation, giving the determinant $|a|$ of the theorem to be proved:

$$
|a|=R\left(u_{1}\right), R\left(u_{2}\right), \cdots, R\left(u_{n}\right)|w| .
$$

For $|a|$, and accordingly $|w|$, to be zero, it is necessary and sufficient that the equations (5), subject to the conditions (4), admit a solution $\left(c_{1}, \cdots, c_{n}\right)$ in which the $c_{i}$ 's are not all zero. That is, for $|w|$ and $|a|$ to be zero, it is necessary and sufficient that $\left(u_{0}, 0\right)$ be conjugate to $\left(u_{n+1}, 0\right)$.

It remains to prove that if a is of rank less than $n$, its rank is exactly $n-1$. Suppose $|a|=0$. Then $\left(u_{0}, 0\right)$ is conjugate to $\left(u_{n+1}, 0\right)$. The point $\left(u_{0}, 0\right)$ cannot also be conjugate to $\left(u_{n}, 0\right)$, for otherwise $\left(u_{n}, 0\right)$ would be conjugate to $\left(u_{n+1}, 0\right)$, contrary to the original choice of $\left(u_{1}, \cdots, u_{n}\right)$. Now a consideration of the form of the minor $A_{n-1}$ of the element $a_{n n}$ of a shows that the vanishing of $A_{n-1}$ is the condition that $\left(u_{0}, 0\right)$ be conjugate to $\left(u_{n}, 0\right)$. Hence $A_{n-1} \neq 0$, and a is of rank $n-1$. Thus the theorem is completely proved.

We denote by $A_{i}(i=1,2, \cdots, n)$ the determinant obtained from a by striking out the last $n-i$ rows and columns of a, and set $A_{0}=1$.

Corollary 1. A necessary and sufficient condition that $A_{i}=0 \quad(i=1$, $2, \cdots, n)$ is that $\left(u_{0}, 0\right)$ be conjugate to $\left(u_{i+1}, 0\right)$.

This follows at once from the form of $A_{i}$ and from Theorem I.

COROLLARY 2. The matrix a is in normal form.*

For if $A_{r}$ and $A_{r+1}(0<r<n)$ were both zero, $\left(u_{0}, 0\right)$ would be conjugate

* Bocher, Introduction to Higher Algebra, p. 59. 
to both $\left(u_{r}, 0\right)$ and $\left(u_{r+1}, 0\right)$, contrary to the original restrictions on $\left(u_{1}\right.$, $\left.u_{2}, \cdots, u_{n}\right)$.

6. The type number of the extremal segment $g$. We prove the following theorem:

Theorem 2.* If the final end point $B$ of the extremal segment $g$ of $\$ 1$ is not conjugate to its initial point $A$, but there are $k$ points $(0 \leqq k \leqq n)$ on $g$ between $A$ and $B$ conjugate to $A$, then the symmetric quadratic form

$$
\sum_{i j} J_{v_{i} v_{j}} v_{i} v_{j} \quad(i, j=1,2, \cdots, n),
$$

when reduced by a real non-singular linear transformation to squared terms only, will have $k$ coefficients that are negative, and $n-k$ that are positive.

The number $k$ is called the type number of the critical point $(0, \cdots, 0)$ of $J\left(v_{1}, \cdots, v_{n}\right)$, and also the type number of $g$.

To prove this theorem we shall make use of the well known fact (Bôcher, loc. cit., p. 147) that in a regularly arranged quadratic form the type number $k$ equals the number of changes of sign in the sequence $A_{0}, A_{1}, \cdots, A_{n}(\$ 5)$ where an $A_{i}$ which is zero is counted as positive or negative at pleasure. To continue we adopt the method of mathematical induction. In the case $n=1$ we are concerned simply with the fixed end points $\left(u_{0}, 0\right)$ and $\left(u_{2}, 0\right)$, and an intermediate point $\left(u_{1}, 0\right)$. Here

$$
A_{1}=\left[w_{0,1}^{\prime}\left(u_{1}\right)-w_{2,1}^{\prime}\left(u_{1}\right)\right] R\left(u_{1}\right) .
$$

It is readily seen that this difference is negative or positive according to whether or not $\left(u_{0}, 0\right)$ possesses a conjugate point prior to $\left(u_{2}, 0\right)$. We now distinguish between the cases in which $A_{n-1}=0$, and $A_{n-1} \neq 0$.

Case I. $A_{n-1} \neq 0$. If we assume the validity of the theorem for the case where there are $n-1$ coördinates $v_{1}, \cdots, v_{n-1}$, we can conclude that there are as many changes in sign in the sequence $A_{0}, \cdots, A_{n-1}$ as there are conjugate points prior to $\left(u_{n}, 0\right)$. To determine whether there is a change of sign in passing from $A_{n-1}$ to $A_{n}$, it is useful to consider again equations (5) of $\$ 5$, unaltered except that the zero in the right hand member of the last equation is here replaced by

$$
A_{n-1} / A_{n}
$$

and the left hand member of the $i$ th one of these equations is here to be multiplied by $R\left(u_{i}\right)(i=1,2, \cdots, n)$.

\footnotetext{
* The corresponding theorem in $m$ dimensions has recently been discovered by the author.
} 
The resulting equations can now be solved for the constants $c_{1}, \cdots, c_{n}$. In particular we obtain, by Cramer's rule,

$$
c_{n}=\frac{A_{n-1}^{2}}{A_{n}^{2}}>0 .
$$

The curve $\lambda$ of $\$ 5$ corresponding to these constants $\left(c_{1}, \cdots, c_{n}\right)$ has just one corner, and that at $\left(u_{n}, c_{n}\right)$. The last equation of (5), $\$ 5$, altered as we have said, now tells us that at this corner the slope of the first segment of $\lambda$ exceeds or is less than the slope of the second segment of $\lambda$, according as the right hand member (3) is positive or negative. This result can be interpreted in terms of the J. D. E. to mean that there is, or is not, a conjugate point of $\left(u_{0}, 0\right)$ between $\left(u_{n}, 0\right)$ and $\left(u_{n+1}, 0\right)$, according as the quotient (3) is negative or positive. Thus, in case $A_{n-1} \neq 0$, the total number of changes of sign in the sequence $A_{0}, A_{1}, \cdots, A_{n}$ equals the total number of conjugate points of $\left(u_{0}, 0\right)$ prior to $\left(u_{n+1}, 0\right)$.

Case II. $A_{n-1}=0$. In this case we make use of the fact that, if $A_{n-1}=0$ in a regularly arranged non-singular quadratic form, then $A_{n-2} \neq 0$. Assuming then the validity of the theorem for the case where there are $n-2$ points between the end points of a given extremal we can conclude that there are as many changes of sign, say $h$, in the sequence $A_{0}, A_{1}, \cdots, A_{n-2}$ as there are conjugate points of $\left(u_{0}, 0\right)$ prior to $\left(u_{n-1}, 0\right)$. But since $A_{n-1}=0$ it follows from Corollary $1, \S 5$, that $\left(u_{n}, 0\right)$ is conjugate to $\left(u_{0}, 0\right)$ so that there are altogether $h+1$ conjugate points of $\left(u_{0}, 0\right)$ prior to $\left(u_{n+1}, 0\right)$. On the other hand it follows from the theory of regularly arranged quadratic forms that if $A_{n-1}=0$, then $A_{n-2}$ and $A_{n}$ have opposite signs. Thus in the sequence $A_{0}$, $A_{1}, \cdots, A_{n}$, there are $h+1$ changes of sign, and the theorem is proved in Case II as well as in Case I.

Part II. Relations in the large between extremals joining $A$ to $B$

7. The integrand and region $S$. In the developement of this chapter we do not wish to exclude the case where the end points of the extremal segment are conjugate. The complete treatment of an extremal segment whose ends are conjugate, both in our theory and in the classical theory, depends upon the nature of the singularity at $B$ of the envelope of the extremals passing through $A$. Such a treatment, at least as developed so far, requires the assumption that the functions used be analytic.

I. We therefore assume that the function $F(x, y, \dot{x}, \dot{y})$ is positively homogeneous of the first degree in $\dot{x}$ and $\dot{y}$, and analytic in all of its arguments, for $x, y$ any point interior to an open region $S$ of the $(x, y)$ plane, and $\dot{x}$ and $\dot{y}$ any 
two numbers not both zero. We shall also assume that for the same arguments

$$
F_{1}(x, y, \dot{x}, \dot{y})>0 \text {. }
$$

In $S$ the differential equations of the extremals can be put in the Bliss* form,

$$
\frac{d x}{d s}=\cos \theta, \frac{d y}{d s}=\sin \theta, \quad \frac{d \theta}{d s}=H(x, y, \cos \theta, \sin \theta),
$$

where

$$
H(x, y, \dot{x}, \dot{y})=\frac{F_{y \dot{x}}-F_{x \dot{y}}}{F_{1}\left[\dot{x}^{2}+\dot{y}^{2}\right]^{3 / 2}} .
$$

A necessary and sufficient condition that a solution $g$ of $(1)$ on which $(x, y, \theta)$ $=\left(x_{0}, y_{0}, \theta_{0}\right)$ for some value of $s$, be identical as a set of points $(x, y)$, with the solution on which $(x, y, \theta)=\left(x_{0}, y_{0}, \theta_{0}+\pi\right)$ for some value of $s$, is that, on $g$,

$$
H(x, y, \cos \theta, \sin \theta)+H(x, y,-\cos \theta,-\sin \theta)=0 .
$$

II. $\dagger$ We assume that (3) holds identically for every point $(x, y)$ in $S$, and for every $\theta$. This type of problem is termed reversible.

8. The regions $S_{1}$, and $R$. We can choose between a great variety of assumptions that will serve as boundary conditions. The following perhaps are as simple as any.

III. Let there be given a closed region $S_{1}$, consisting of points interior to $S$, bounded by a simple closed curve $\beta$ consisting of a finite number of analytic arcs. We assume that this boundary $\beta$ is extremal-convex $\ddagger$, in the sense that the interior angles at the vertices of $\beta$ shall be between 0 and $\pi$, and that an extremal tangent to an analytic arc $\beta^{\prime}$ of $\beta$ at a point $P$, shall in the neighborhood of $P$, except for $P$, lie wholly on that side of $\beta^{\prime}$ which is exterior to $S_{1}$.

IV. + We assume further that the region $S_{1}$ is covered in a one-to-one manner by a proper field of extremals of the form

$$
x=h(u, v), \quad y=k(u, v),
$$

where $u$ is the parameter, and $v$ is the arc length measured along the extremals, where at every point $(u, v)$ that corresponds to a point $(x, y)$ in $S_{1}$ the functions (1) are single-valued and analytic in $u$ and $v$, and

* Bliss, these Transactions, vol. 7 (1906), p. 180.

† The author has recently removed hypotheses II and IV, assuming then that $F$ is positive definite. Powerful results obtain, but the proofs are necessarily more difficult.

† Compare Bolza, loc. cit., pp. 276-278, and also Birkhoff, loc. cit., pp. 216-219. 


$$
D=\left|\begin{array}{ll}
h_{u} & h_{v} \\
k_{u} & k_{v}
\end{array}\right| \neq 0 .
$$

The region $S_{1}$ in the $(x, y)$ plane will correspond in the $(u, v)$ plane to a closed region $R$, bounded by a simple closed curve $\gamma$ consisting of a finite number of analytic arcs, and again extremal-convex. When $u$ is the independent variable, and $v$ the dependent variable, the integral $J$ can be put in the non-parametric form as in $\$ 3$ with $f\left(u, v, v^{\prime}\right)$ the integrand. Because of the assumption of reversibility the non-parametric problem, with integrand $f\left(u, v, v^{\prime}\right)$, will here include all the extremals of the parametric form except those of the family $u=$ constant. From our assumption regarding $F_{1}$, we have here that

$$
f_{v^{\prime} v^{\prime}}\left(u, v, v^{\prime}\right)=0,
$$

for all points $(u, v)$ in $R$, and any number $v^{\prime}$. Concerning the region $R$, we can now establish the following lemma:

LEMMA. Let $a$ and $b$ be, respectively, the minimum and maximum values of $u$ on $\gamma$. Then $\gamma$ consists of two arcs of the form

$$
v=A(u), v=B(u), \quad a \leqq u \leqq b,
$$

where $A(u)$ and $B(u)$ are of class $C$, and analytic in $u$ on the interval $a \leqq u \leqq b$, except for a finite set of values of $u$, while

$$
A(u)<B(u), \quad a<u<b,
$$

Finally the interior points $(u, v)$ of $R$ are the points satisfying

$$
A(u)<v<B(u), \quad a<u<b .
$$

To prove these statements let $\left(u_{0}, v_{0}\right)$ be any interior point of $R$. There exist two numbers, $v_{1}$ and $v_{2}$, of such sort that the points $(u, v)$ for which

$$
u=u_{0},
$$$$
v_{1}<v<v_{2},
$$

include the point $\left(u_{0}, v_{0}\right)$, but no points other than interior points of $R$, while the points $\left(u_{0}, v_{1}\right)$ and $\left(u_{0}, v_{2}\right)$ are on the boundary of $R$. Now the extremal-segment (5) is not tangent to $\gamma$ at either of its ends, since $\gamma$ is extremal convex. If $\gamma$ has a vertex at $\left(u_{0}, v_{2}\right)$, the two analytic arcs adjoining $\left(u_{0}, v_{2}\right)$ must lie on opposite sides of $u=u_{0}$, at least in the neighborhood of $\left(u_{0}, v_{2}\right)$, for otherwise the points of $(5)$ in the neighborhood of $\left(u_{0}, v_{2}\right)$ would not lie in $R$. A similar statement applies to $\left(u_{0}, v_{1}\right)$. Thus, in 
any case, there must exist functions $h(u)$ and $k(u)$ of class $C$, and constants $a_{1}$ and $b_{1}$, differing from $u_{0}$ by so little, that the points $(u, v)$ satisfying

$$
h(u)<v<k(u),
$$

$$
a_{1}<u<b_{1},
$$

are all interior points of $R$, while the curves

$$
\begin{array}{lll}
v=h(u), & v_{1}=h\left(u_{0}\right), & a_{1} \leqq u \leqq b_{1}, \\
v=k(u), & v_{2}=k\left(u_{0}\right), & a_{1}<u_{0}<b_{1},
\end{array}
$$

lie on the boundary of $R$.

We wish to show that these functions $h(u)$ and $k(u)$ can be extended in definition, as functions of class $C$, so that the preceding statements regarding (6) and (7) still hold true when $a_{1}=a$ and $b_{1}=b$. Whether this is true or not there certainly exist constants $a_{2}$ and $b_{2}$, such that

$$
a \leqq a_{2}<u_{0}<b_{2} \leqq b,
$$

and functions $h(u)$ and $k(u)$ of class $C$, such that the curves

$$
\begin{aligned}
& v=h(u), \quad v_{1}=h\left(u_{0}\right), \\
& v=k(u), \quad v_{2}=k\left(u_{0}\right), \quad a_{2} \leqq u \leqq b_{2},
\end{aligned}
$$

lie on the boundary of $R$, and the points $(u, v)$ which satisfy

$$
h(u)<v<k(u), \quad a_{2}<u<b_{2},
$$

are all interior points of $R$, while further, $a_{2}$ and $b_{2}$ are constants such that the interval in (8), and the corresponding interval in (9), cannot be expanded at either or both ends, and the preceding statements about (8) and (9) still hold true for these expanded intervals.

The following division into cases is exhaustive:

\section{Case I}

Case II

Case III

Case IV

$$
\begin{aligned}
a_{2}=a, & b_{2}=b ; \\
a_{2}=a, & b_{2}<b ; \\
a<a_{2}, & b_{2}=b ; \\
a<a_{2}, & b_{2}<b .
\end{aligned}
$$

Case I. In this case the theorem follows readily.

Case II. In this case we will prove that the inequality $b_{2}<b$ is impossible. In the first place if we had $h\left(b_{2}\right)=k\left(b_{2}\right)$, the curve (8) would completely bound the region (9), a sub-region of $R$. This is impossible, since $R$ consists of a single connected region. Hence $h\left(b_{2}\right)<k\left(b_{2}\right)$. The points $(u, v)$ which satisfy

$$
h\left(b_{2}\right)<v<k\left(b_{2}\right), \quad u=b_{2},
$$


are limit points of points of (9), and are accordingly points of $R$. We distinguish again between two cases, Cases IIa and IIb.

In Case IIa we suppose the points of (10) are all interior points of $R$. In this case (8) and (9) will hold as stated for a slightly larger $b_{2}$, and thus we have a contradiction.

In Case IIb we suppose the points of (10) include at least one boundary point $P$ of $R$. Such a point $P$ cannot be a vertex of the boundary without the interior angle at the vertex being greater than $\pi$, contrary to an hypothesis. Neither can $P$ be an ordinary point of the boundary, for in that case the boundary would have to be tangent to the extremal segment (10), a result which is again impossible, since the boundary is extremal-convex. Thus Case IIb leads to a contradiction under all circumstances, and is impossible.

Similarly, it can be proved that Cases III and IV are impossible. Case I alone is possible, and the theorem in italics follows readily.

9. Further properties of the region $R$. In $R$ no extremals other than the extremals $u=$ constant can be tangent to the extremals $u=$ constant. Hence every extremal segment other than the extremal segments $u=$ constant is representable in the form $v=M(u)$, where $M(u)$ is an analytic function of $u$, for $u$ on the interval ( $\$ 8$, Lemma)

$$
a \leqq u \leqq b,
$$

or some sub-interval of that interval.

Let $A$ and $B$ be two distinct points of $R$, either interior to $R$, or on the boundary of $R$. Any extremal joining $A$ to $B$ in $R$ will have no points on the boundary of $R$, except possibly its end points $A$ or $B$. This follows readily from the extremal convex nature of the boundary $(\$ 8)$.

We will now prove that two points $A$ and $B$ in $R$ can be joined in $R$ by at most a finite number of extremal segments. If $A$ and $B$ are both on the same extremal segment $u=u_{0}$, that extremal segment is the only extremal segment in $R$ which can join $A$ to $B$. Suppose then that $\left(u_{0}, v_{0}\right)$ and $\left(u_{1}, v_{1}\right)$ are two points of $R$ for which $u_{0}<u_{1}$, and which can be joined by an infinite set of extremal segments in $R$. Denote by $m$ the angles in the $(u, v)$ plane which the tangents to these extremals at $\left(u_{0}, v_{0}\right)$ make with a parallel to the positive $u$ axis. Take these angles between $-\pi / 2$ and $\pi / 2$.

Now there will be at least one limit angle $m_{0}$ of these angles $m$. The $v$ coördinate of an extremal $E_{0}$ issuing from $\left(u_{0}, v_{0}\right)$ with the angle $m_{0}$ can be continued as an analytic function of $u$ until $E_{0}$ passes out of $R$ or through $\left(u_{1}, v_{1}\right)$. The extremal $E_{0}$ cannot pass out of $R$ before passing through $\left(u_{1}, v_{1}\right)$, because if $E_{0}$ did so pass out of $R$, extremals with angles $m$ sufficiently 
near $m_{0}$ would also pass out of $R$ before passing through $\left(u_{1}, v_{1}\right)$, which is impossible.

It would follow then from the envelope theory of extremals that all extremals, without exception, that issue from $\left(u_{0}, v_{0}\right)$ with angles neighboring $m_{0}$ would pass through $\left(u_{1}, v_{1}\right)$. Moreover, we could then prove that all extremals issuing from $\left(u_{0}, v_{0}\right)$ with angles $m$ for which

$$
m_{0} \leqq m<\pi / 2 \text {, }
$$

would remain in $R$, at least until they passed through $\left(u_{1}, v_{1}\right)$. For otherwise there would be a least upper bound $m_{1}<\pi / 2$, of angles $m$ at which extremals in $R$ issue from $\left(u_{0}, v_{0}\right)$ and pass through $\left(u_{1}, v_{1}\right)$. But the extremal issuing from $\left(u_{0}, v_{0}\right)$ with the angle $m_{1}$ would lie entirely within $R$, except possibly for its end points, so that extremals issuing from $\left(u_{0}, v_{0}\right)$ with angles slightly larger than $m_{1}$ would also lie in $R$, and pass through $\left(u_{1}, v_{1}\right)$. Therefore no such least upper bound, $m_{1}$, for which $m<\pi / 2$ exists. Thus all extremals which issue from $\left(u_{0}, v_{0}\right)$ with angles $m$ satisfying (1) must pass through $\left(u_{1}, v_{1}\right)$ before passing out of $R$.

But an extremal issuing from $\left(u_{0}, v_{0}\right)$ with an angle sufficiently near $\pi / 2$ will pass out of $R$ without passing through $\left(u_{1}, v_{1}\right)$. From this contradiction we infer the truth of the statement to be proved.

10. The function $J\left(v_{1}, \cdots, v_{n}\right)$. None of the extremal segments $u=u_{0}$ in $R$ have a point on them conjugate to either end point. To prove this let the J. D. E. in the Weierstrass form with independent variable $t=v$, be set up corresponding to the extremal $u=u_{0}$ in the $(x, y)$ plane. This differential equation has as a solution the determinant $D$ of $\S 8$, provided we set $u=u_{0}$ and let $v$ vary. The absence of any conjugate points on $u=u_{0}$ follows from the non-vanishing of this determinant. From the absence of conjugate points in $R$ on the extremals $u=u_{0}$, the "regularity" $\left(F_{1}>0\right)$, and analyticity of the problem, and the extremal convex nature of the boundary, it follows that a positive constant e can be determined small enough to have the following properties. Any point of $R$ for which $u=u_{0}$, can be joined to any point of $R$ for which $u$ differs from $u_{0}$ in absolute value by less than e, by a unique analytic extremal $h$ lying entirely within $R$, excepting possibly its end points, and such that on $h$ there are no pairs of conjugate points (B, p. 307). The questions of uniformity arising can be met by the reader without too much difficulty.

Now let there be given two points $A$ and $B$ of $R$, not on the same extremal segment $u=u_{0}$. We are concerned with the extremals joining $A$ to $B$, if any such exist. Let $u_{0}, u_{1}, \cdots, u_{n+1}$ be a set of increasing values of $u$ of which successive values differ at most by the constant $e$ of the preceding paragraph, and which are such that $A$ lies on $u=u_{0}$, and $B$ on $u=u_{n+1}$. Consistent with 
this, let the coördinates of $A$ and $B$ be respectively $\left(u_{0}, v_{0}\right)$ and $\left(u_{n+1}, v_{n+1}\right)$, and $\left(v_{1}, \cdots, v_{n}\right)$ be variables such that the points

$$
\left(u_{1}, v_{1}\right), \cdots,\left(u_{n}, v_{n}\right)
$$

are all in $R$. Let the successive points of the set

$$
\left(u_{0}, v_{0}\right),\left(u_{1}, v_{1}\right), \cdots,\left(u_{n+1}, v_{n+1}\right)
$$

be joined by the unique extremal segments of the preceding paragraph, and let the integral $J$ be evaluated along the resulting curve. If we hold the end points $A$ and $B$ fast, as well as the $u$ coördinates of the intermediate points, the value of $J$ will be a function, $J\left(v_{1}, \cdots, v_{n}\right)$, that will be analytic in $\left(v_{1}, \cdots, v_{n}\right)$ in the domain (Lemma $\S 8$ )

$$
A\left(u_{i}\right) \leqq v_{i} \leqq B\left(u_{i}\right) \quad(i=1,2, \cdots, n) .
$$

The partial derivatives of the function $J$ are given by the formulas

$$
J_{v_{i}}\left(v_{1}, \cdots, v_{n}\right)=f_{v^{\prime}}\left(u_{i}, v_{i}, p_{i}\right)-f_{v^{\prime}}\left(u_{i}, v_{i}, q_{i}\right) \quad(i=1,2, \cdots, n),
$$

where $p_{i}$ is the slope at $\left(u_{i}, v_{i}\right)$ of the extremal joining $\left(u_{i-1}, v_{i-1}\right)$ to $\left(u_{i}, v_{i}\right)$, and $q_{i}$ is the slope at $\left(u_{i}, v_{i}\right)$ of the extremal joining $\left(u_{i}, v_{i}\right)$ to $\left(u_{i+1}, v_{i+1}\right)$. Since

$$
f_{v^{\prime} v^{\prime}}\left(u, v, v^{\prime}\right)>0
$$

for every $(u, v)$ in $R$ and every $v^{\prime}$, the partial derivative (4) is zero, when and only when $p_{i}=q_{i}$. We have the result that the function $J\left(v_{1}, \cdots, v_{n}\right)$ has a critical point $\left(v_{1}, \cdots, v_{n}\right)$, when and only when the points (2) all lie on a single analytic extremal.

11. Relations between critical points. A lemma fundamental for our present purposes is derived from the Corollary to Theorem 8, page 392 of the paper of the author's already cited. Before stating the lemma let it be agreed that the positive normal to an analytic boundary of a region $\Sigma$ will be understood to be that sensed normal that leads from points in $\Sigma$ to points without $\Sigma$.

LEMMA 1. Let there be given a closed region $\Sigma$ in the space of the $n$ variables $\left(x_{1}, \cdots, x_{n}\right)$, bounded by a closed analytic manifold, without singularity and homeomorphic with the interior and boundary of an (n-1)-dimensional hypersphere. In $\Sigma$ let there be given a function $f\left(x_{1}, \cdots, x_{n}\right)$, of class $C^{\prime \prime \prime}$ at each point of $\Sigma$, and possessing on the boundary of $\Sigma$ a normal directional derivative that is positive. Suppose the critical points of $f\left(x_{1}, \cdots, x_{n}\right)$ are all of rank $n$. Of the type numbers $k$ of these critical points, let $m$ be the maximum. Let $M_{k}$ be the number of critical points of type $k$. Then between the integers $M_{k}$ the following relations hold: 
$1 \leqq M_{0}$,

$1 \geqq M_{0}-M_{1}$,

$1 \leqq M_{0}-M_{1}+M_{2}$,

(R) $1 \geqq M_{0}-M_{1}+M_{2}-M_{3}$,

$1 \leqq\left[M_{0}-M_{1}+M_{2}-M_{3}+-\cdots+(-1)^{m-1} M_{m-1}\right](-1)^{m-1}$,

$1=M_{0}-M_{1}+M_{2}-M_{3}+-\cdots+(-1)^{m} M_{m}$.

In terms of the functions of the Lemma, §8, we can say that the domain of the points $\left(v_{1}, \cdots, v_{n}\right)=(V)$ for which the function $J\left(v_{1}, \cdots, v_{n}\right)$ of $\$ 10$ is defined, is a rectangular hyperspace made up of points $(V)$ satisfying the inequalities

$$
A\left(u_{i}\right) \leqq v_{i} \leqq B\left(u_{i}\right) \quad(i=1,2, \cdots, n),
$$

where the numbers $\left(u_{1}, \cdots, u_{n}\right)$ are constants specified in $\$ 10$. The boundary of the domain obviously consists of hyperrectangles on $2 n$ hyperplanes

taken separately.

$$
v_{i}=A\left(u_{i}\right), \quad v_{i}=B\left(u_{i}\right) \quad(i=1,2, \cdots, n),
$$

To apply Lemma 1 of this section we need to find the normal directional derivative of $J\left(v_{1}, \cdots, v_{n}\right)$ at points on the boundary of (1).

Let $\left(a_{1}, a_{2}, \cdots, a_{n}\right)$ be a point $(V)$ of (1) for which $a_{1}=A\left(u_{1}\right)$, that is, a point on the bounding hyperplane, $v_{1}=A\left(u_{1}\right)$. If we hold the variables $\left(v_{2}, \cdots, v_{n}\right)$ constantly equal to $\left(a_{2}, \cdots, a_{n}\right)$, respectively, and let $v_{1}$ decrease so as to pass through $v_{1}=a_{1}$, the corresponding point $\left(v_{1}, a_{2}, \cdots\right.$, $a_{n}$ ) will vary on a normal to the hyperplane $v_{1}=A\left(u_{1}\right)$, and pass out of the domain (1). But the point $\left(v_{1}, \cdots, v_{n}\right)=\left(a_{1}, \cdots, a_{n}\right)$ will correspond (\$10) to a succession of $n+1$ extremal segments joining $A$ to $B$ in $R$. Of these segments the first two will adjoin each other in $R$ at the point $(u, v)=\left(u_{1}, a_{1}\right)$. The partial derivative of $J\left(v_{1}, \cdots, v_{n}\right)$ with respect to $v_{1}$ at the point $\left(a_{1}, \cdots, a_{n}\right)$ can be obtained from (4), $\$ 10$, by putting $i=1$. Using the law of the mean we then obtain the equation

$$
J_{v_{1}}\left(a_{1}, a_{2}, \cdots, a_{n}\right)=f_{v^{\prime} v^{\prime}}\left[u_{1}, a_{1}, \bar{p}\right]\left[p_{1}-q_{1}\right],
$$

where $\bar{p}$ is a number between $p_{1}$ and $q_{1}$. From the extremal-convex nature of the boundary it follows that $p_{1}<q_{1}$. Thus this derivative (2) is negative.

The directional derivative of $J$ along the normal to the hyperplane $v_{1}=A\left(u_{1}\right)$ at the point $\left(a_{1}, a_{2}, \cdots, a_{n}\right)$ is to be taken in the sense that leads out of (1), that is in the sense of decreasing $v_{1}$. This directional derivative is thus equal to the left hand member of (2) multiplied by $(-1)$, and is 
accordingly positive. Similarly the directional derivative of $J$ along a positive normal to any of the other hyperplanes bounding (1) may be seen to be positive.

But we cannot as yet apply the lemma on critical points because the boundary of (1) is made up of portions of $2 n$ hyperplanes instead of one analytic manifold. To meet this difficulty let $O$ be any interior point of (1), and let straight line rays be drawn from $O$ to each point of the boundary of (1). It follows from the results of the preceding paragraph that the directional derivative of $J\left(v_{1}, \cdots, v_{n}\right)$, at a point $P$ on the boundary of (1), taken along the ray joining $O$ to $P$ in the sense that leads from $O$ to $P$ is always positive. Now the domain (1) can be projectively transformed into an $n$-dimensional hypercube that lies in the space of $\left(y_{1}, \cdots, y_{n}\right)$, and that is bounded by the $(n-1)$-dimensional hyperplanes

$$
y_{i}= \pm 1 \quad(i=1,2, \cdots, n) .
$$

This hypercube can be approximated to by the analytic manifold

$$
y_{1}^{2 r}+y_{2}^{2 r}+\cdots+y_{n}^{2 r}=1,
$$

where $r$ is a positive integer. In fact, if $e$ be a positive constant, it is easy to show that for $r$ sufficiently large, points on the above hypercube and the manifold (4) that lie on the same ray issuing from the origin will be within a distance $e$ of one another.

Let now the hypercube be projectively transformed back into (1), and suppose the manifold (4) goes into a manifold $M$. Let the point $O$ from which rays were drawn in (1) be the image of the origin in the hypercube. The rays in (1) will each meet the manifold $M$ in a single point. If $r$ be sufficiently large, $M$ will approximate the domain (1) so closely that the directional derivatives of $J$ at points of $M$ on the rays issuing from $O$ in the sense that leads away from $O$, will all be positive. It follows that the outer normal directional derivatives at points of $M$ will be positive.

A second requirement on $M$ is that it approximate the domain (1) so closely that it contain in its interior all the critical points of $J\left(v_{1}, \cdots, v_{n}\right)$ that (1) contains. The manifold $M$ will serve as the manifold $\Sigma$ of Lemma 1 of this section.

The critical points of Lemma 1 are of rank $n$. For the moment, then, we restrict ourselves to extremals joining $A$ to $B$ on which $A$ is not conjugate to $B$. Reference to Theorem 2 of $\S 6$ and Lemma 1 of this section gives the lemma.

LEMMa 2. Let there be given regions $S$ and $S_{1}$, and integrand $F$, satisfying the hypotheses I, II, III, and IV, of $\$ 7$ and $\S 8$. In $S_{1}$ let there be given two 
points $A$ and $B$ which are joined by no extremals on which $A$ is conjugate to $B$. Let the number of extremals joining $A$ to $B$ in $S_{1}$ on which there are $k$ conjugate points of $A$ prior to $B$ be denoted by $M_{k}$. Let $m$ be an integer equal to the maximum of these integers $k$. Then between the numbers $M_{k}$ the relations (R) of Lemma 1 hold.

12. A theorem in the large. We seek now to remove from Lemma 2, $\$ 11$, the restriction that on no extremal joining $A$ to $B$ in $S_{1}$ is $A$ conjugate to $B$. Before doing this it will be necessary to recall certain results obtained by Lindeberg.*

Let there be given in $R$ an analytic extremal $E_{0}$ of the form

$$
v=E(u), \quad c \leqq u \leqq d,
$$

joining $A$ to $B$, and on which the point at which $u=c$ has for its $(m+1)$ st conjugate point the point at which $u=d$. Let $\alpha$ be the slope at $A$ of any extremal through $A$. In particular let $\alpha=\alpha_{0}$ be the slope of $E_{0}$ at $A$. That part of the envelope of the family of extremals through $A$ which lies in the neighborhood of $B$, will not here consist merely of the point $B$. For according to the envelope theory this could only happen if all the extremals through $A$ with slopes $\alpha$ near $\alpha_{0}$ should pass through $B$, contrary to results in $\$ 9$.

According to Lindeberg, the envelope $T$ of the family of extremals passing through $A$, with a slope $\alpha$ near $\alpha_{0}$, will then be of the form

$$
\begin{aligned}
v-E(u) & =\left(\alpha-\alpha_{0}\right)^{r+1} K(\alpha), \quad r>0, & & K\left(\alpha_{0}\right) \neq 0, \\
u-d & =\left(\alpha-\alpha_{0}\right)^{r} H(\alpha), & & H\left(\alpha_{0}\right) \neq 0,
\end{aligned}
$$

where $K(\alpha)$ and $H(\alpha)$ are analytic in $\alpha$ at $\alpha=\alpha_{0}$, and $r$ is a positive integer.

Three classes of envelopes can be distinguished:

Class I $r$ is odd;

Class II $r$ is even and $H\left(\alpha_{0}\right)<0$;

Class III $r$ is even and $H\left(\alpha_{0}\right)>0$.

Class I. Here the envelope $T$ is tangent to the extremal $E_{0}$ at $B$, has no cusp there, and lies wholly on one side of $E_{0}$ near $B$. If the class of extremals through $A$ be restricted to extremals $E$ for which $\left|\alpha-\alpha_{0}\right|$ is sufficiently small, we can say that through each point $P$, not on $T$, but sufficiently near $B$, and on the same side of $T$ as $E_{0}$, there will pass just two extremals of the set $E$. On these two extremals the type number $k$, that is, the number of points which are conjugate to $A$ and prior to $P$, will equal $m$ and $m+1$,

* Lindeberg, Mathematische Annalen, vol. 59 (1904), p. 321. 
respectively. Through any point $P$, not on $T$, but sufficiently near $B$ and on the opposite side of $T$ from $E_{0}$, there will pass no extremals of the set $E$.

Class II. Here $T$ is tangent to the extremal $E_{0}$ at $B$, but has a cusp there. On the envelope near $B, u<d$, except for the point $B$. The two branches of the cusp lie on opposite sides of $E_{0}$. A point $P$ sufficiently near $B$, within the cusp, but not on $T$, can be joined to $A$ by three extremals neighboring $E_{0}$. On these three extremals the type numbers $k$ will have the values $m+1$, $m+1$, and $m$, respectively. Through any point $P$ sufficiently near $B$, without the cusp, but not on $T$, there passes just one extremal issuing from $A$ and neighboring $E_{0}$. On this extremal $k=m+1$.

Class III. Here $T$ is tangent to the extremal $E_{0}$ at $B$, but has a cusp there. On the envelope near $B, u>d$, except for the point $B$. The two branches of the cusp lie on opposite sides of $E_{0}$. A point $P$, sufficiently near $B$, within the cusp, but not on $T$, can be joined to $A$ by three extremals neighboring $E_{0}$. On these three extremals $k$ has the values $m, m$, and $m+1$, respectively. Through each point $P$ sufficiently near $B$, without the cusp, and not on $T$, there passes just one extremal issuing from $A$ and neighboring $E_{0}$. On this extremal $k=m$.

In the following theorem there are a number of conventions to be adopted. Let $g$ be an extremal joining $A$ to $B$ on which there are $m$ points conjugate to $A$ prior to $B$. If $B$ is not conjugate to $A, g$ is to be counted as one extremal of type $k=m$. If $B$ is conjugate to $A$, and $g$ belongs to Class $\mathrm{I}$, of the preceding classification, $g$ is to be counted as two extremals, of types $k=m+1$ and $k=m$ respectively. If $B$ is conjugate to $A$, and $g$ belongs to Class II, or to Class III, then $g$ is to be counted as one extremal of type $k=m+1$, or of type $k=m$, respectively. We can now prove the following theorem "in the large."

THEOREM 3. Let there be given regions $S$ and $S_{1}$ and an integrand $F$ satisfying hypotheses I, II, III, and IV, of $\S 7$ and §8. Let $A$ and $B$ be any two points of $S_{1}$. A first conclusion is that there are at most a finite number of extremals $g$ joining $A$ and $B$ and lying in $S_{1}$. Let $M_{k}$ be the number of these extremals $g$ of type $k$, counted according to the conventions preceding this theorem, and let $m$ be the maximum of these integers $k$. Then between the numbers $M_{k}$ the relations (R) of Lemma 1, 11 , hold true.

That there are at most a finite number of extremals $g$ joining $A$ to $B$ in $S_{1}$, was proved in $\S 9$. If on no extremal $g, A$ is conjugate to $B$, the remainder of the theorem follows from Lemma $2, \S 11$.

If there are a number of extremals $g$ on which $A$ is conjugate to $B$, each of these extremals $g$ will be tangent at $B$ to an envelope $T$ of extremals issuing from $A$ and neighboring that $g$. Let $L$ be a short straight line segment 
passing through $B$, lying in $S_{1}$, and tangent to none of these envelopes. Any point $P$ on $L$, not $B$, but sufficiently near $B$, will lie on none of these envelopes. Concerning the possibility of joining $A$ to $P$ by extremals $g^{\prime}$ in $S_{1}$, we can state the following:

Corresponding to any extremal $g$ on which $A$ is not conjugate to $B$ there will exist one extremal $g^{\prime}$ neighboring $g$, joining $A$ to $P$, and of the same type as $g$.

If $g$ be an extremal on which $A$ is conjugate to $B$, and $g$ is of Class II, the point $P$ if sufficiently near $B$, will lie without the corresponding cusp. According to the preceding description of Class II there will then be just one extremal $g^{\prime}$ joining $A$ to $P$ and neighboring $g$. On $g^{\prime}, A$ will not be conjugate to $P$, and the number of conjugate points of $A$ prior to $P$ will equal the type number $k$ which we have agreed to assign to $g$. The facts are similar for extremals $g$ of Class III.

If $g$ be any extremal of Class $\mathrm{I}$, joining $A$ to $B$, let $T$ be the corresponding envelope neighboring $B$. We consider two cases. In Case I we suppose $P$ lies on the same side of $T$ as $g$. In this case $A$ and $P$ can be joined by two extremals neighboring $g$, on which $A$ is not conjugate to $P$, and whose type numbers are the numbers we have agreed to assign to $g$. In Case II we suppose $P$ lies on the opposite side of $T$ from $g$. In this case there will exist no extremals neighboring $g^{\prime}$ passing from $A$ to $P$. Relative to this case we observe that if the relations (R) of Lemma $1, \S 11$, hold between any given set of integers $M_{k}$, these relations will also hold if we replace $M_{i}$ and $M_{i-1}$ by $M_{i}+1$ and $M_{i-1}+1$, for any particular $i(i=1,2, \cdots, n)$. Finally, if $P$ be sufficiently near $B$, there will be no other extremals $g^{\prime}$ joining $A$ to $P$ than those just enumerated. For if there were more, we could prove by a limiting process that there would be other extremals $g$ joining $A$ to $B$ besides those first supposed to exist.

The extremals which we have just proved join $A$ and $P$ are all extremals on which $A$ is not conjugate to $P$. Concerning them Lemma 1 of $\$ 11$ holds. If there are no extremals $g$ joining $A$ to $B$, of Class I, Case II, the theorem follows directly. But if there are extremals $g$ of Class I, Case II, the relations of Lemma 1, $\S 11$, hold if all extremals $g$ be counted except those of Class I, Case II. As we have already noted, the counting of extremals of Class I, Case II, as if they were in Class I, Case I, will not alter the validity of the relations (1) of $\S 11$, if these relations hold true prior to such a change. Thus the theorem is proved.

Note. We could prove, by the methods of the paper on critical points, that extremals $g$ of. Class I could be omitted from the count entirely, and the relations (R), §11, still hold true. Such a proof would, however, lead us too far astray. 
Another important question is whether there are relations between the numbers $M_{k}$ other than those affirmed in the Theorem. For the case of functions in general, apart from the calculus of variations, the answer is that there are no other relations without further hypotheses. More specifically the author has proved that if there be given any set of integers $M_{i}$ satisfying the relations $(\mathrm{R})$ of Lemma $1, \S 11$, there exists a function $f$ of class $C^{\prime \prime}$ within and on a unit (n-1)-sphere, which on this $(n-1)$-sphere satisfies the boundary conditions of Lemma 1 , and which possesses for each $i, M_{i}$ critical points of type $i$, and no other critical points of any sort.*

For the case of the calculus of variations the author has shown that if under the hypotheses of Theorem 3 there is more than one extremal joining $A$ and $B$, then there are at least two such extremals of minimum type. Except for this, Dr. Richmond, National Research Fellow at Harvard, has recently shown, for the case where $m<3$ in the Theorem, that an example can be set up in the calculus of variations corresponding to any set of integers $M_{i}$ satisfying the relations $(\mathrm{R})$.

13. Existence of extremal-convex boundaries. Let there be given in the $(x, y)$ plane an open or closed curve $\gamma$, without multiple points, and made up of a finite number of arcs of class $C^{\prime}$. If $\gamma$ is closed, the points neighboring $\gamma$ and not on $\gamma$ make up two disconnected regions. If $\gamma$ is open, the points neighboring $\gamma$, not on $\gamma$, and lying on short perpendiculars to the component arcs of $\gamma$, slightly extended at the vertices, again make up two distinct regions. These two disconnected regions will be called the sides of $\gamma$.

The curve $\gamma$ will be said to be extremal-convex relative to one of its sides $S$, if the angles at its vertices on the side $S$ are between 0 and $\pi$, and if an extremal tangent to $\gamma$ at any point $P$ has no other point than $P$ in common with $\gamma$ or $S$ in the neighborhood of $P$.

Let $g$ be any open or closed curve of class $C^{\prime}$ and without multiple points. A curve $g^{\prime}$ of class $C^{\prime}$ will be said to lie arbitrarily near $g$, in position and direction, if corresponding to an arbitrarily small positive constant $e$, a one-to-one continuous correspondence can be set up between $g$ and $g^{\prime}$, in such a fashion that corresponding points are within a distance $e$ of one another, and direction cosines of tangents at corresponding points differ by at most $e$. We can now prove the following lemma:

LEMMA 1. Let $g$ be any extremal segment satisfying the hypothesis in $\$ 1$, and in addition derived from a reversible problem (\$7).

(A) Then there can be found a curve $g_{1}$ of class $C^{\prime \prime \prime}$, arbitrarily near to $g$

- Morse, The analysis and analysis situs of regular $n$-spreads in ( $n+r)$-space, Proceedings of the National Academy of Sciences, vol. 13 (1927), pp. 813-817. 
in position and direction, lying wholly on an arbitrary side of $g$, and such that $g_{1}$ will be extremal-convex relative to the side of $g_{1}$ that does not contain $g$.

(B) If $g$ contains no conjugate point to its end points, then in addition to $g_{1}$ there can also be found a second curve $g_{2}$ with the same properties as $g_{1}$, except that $g_{2}$ will be extremal-convex relative to the side of $g_{2}$ that contains $g$.

To prove (A) we take the problem into the $(u, v)$ plane as in $\$ 2$, so that $g$ becomes a segment of the $u$ axis. The differential equation of the extremals near $g$ can be put in the form

$$
v^{\prime \prime}=A\left(u, v, v^{\prime}\right)
$$

where $A\left(u, v, v^{\prime}\right)$ is of class $C^{\prime \prime \prime}$ for $(u, v)$ neighboring $g$, and any number $v^{\prime}$ sufficiently small in absolute value. Alongside of (1) let us consider the differential equation

$$
v^{\prime \prime}=A\left(u, v, v^{\prime}\right)+M v,
$$

where $M$ is a positive constant such that on $g$

$$
M>A_{v}(u, 0,0) \text {. }
$$

Now $v \equiv 0$ will represent a solution of (2), as well as of (1). The differential equation of the first variation, corresponding to a solution of (2), set up in particular for $g$, on which $v \equiv 0$, will be

$$
w^{\prime \prime}=A_{v^{\prime}}(u, 0,0) w^{\prime}+\left[A_{v}(u, 0,0)+M\right] w .
$$

Let us compare (4), by Sturm's method, with

$$
w^{\prime \prime}=A_{v^{\prime}}(u, 0,0) w^{\prime} .
$$

Since (5) has a solution $w \equiv$ constant $\neq 00,(4)$ has no solution except $w \equiv 0$ which vanishes twice. Hence there is no conjugate point on $g$ to either end point of $g$, relative to the differential equation (2).

Accordingly there exists a curve segment $g_{1}$, which represents a solution of (2), which lies arbitrarily near $g$, and on which $v>0$. A comparison of the value of $v^{\prime \prime}$, say $v_{2}^{\prime \prime}$, given by (2) for a $v$ and $v^{\prime}$ on $g_{1}$, with the value, say $v_{1}^{\prime \prime}$, of $v^{\prime \prime}$ given by (1) for the same $v$ and $v^{\prime}$, shows that $v_{2}^{\prime \prime}>v_{1}^{\prime \prime}$. The proof is similar for the side of $g$ where $v<0$.

To prove (B), compare (1) with

$$
v^{\prime \prime}=A\left(u, v, v^{\prime}\right)-e v^{3},
$$

where $e$ is a positive constant. The equations of first variation corresponding to a solution of (6), or a solution of (1), respectively, are identical when set up for $g$, that is for $v \equiv 0$. Accordingly there is no conjugate point on $g$ 
to any point on $g$, when $g$ is considered as a solution of (6). Hence a curve segment $g_{1}$ exists that represents a solution of (6), that lies arbitrarily near $g$, and on which $v>0$. Part (B) follows on comparing the $v^{\prime \prime}$ given by (6) with that given by (1) for the same $v$ and $v^{\prime}$. Lemma 1 leads to the following lemma.

LEMMA 2. Let there be given in the region $S$ of $\$ 1$ a region $S^{\prime}$ bounded by a simple closed curve $\gamma$ made up of a finite number of extremal segments $g$ of $\$ 1$, at least two in number, and making interior angles between 0 and $\pi$. Suppose further that the problem is reversible.

(A) Then each arc $g$ may be replaced by an arc $g_{1}$ of class $C^{\prime \prime \prime}$, arbitrarily near $g_{1}$, within $S^{\prime}$, and such that the set of arcs $g_{1}$ form a simple closed curve extremal-convex relative to its interior.

(B) If the end points of each arc $g$ have no conjugate points on that arc $g$, each arc $g$ may be replaced by an arc $g_{2}$ of class $C^{\prime \prime \prime}$, arbitrarily near $g_{2}$, exterior to $S^{\prime}$, and such that the set of arcs $g_{2}$ form a simple closed curve extremal-convex relative to its interior.

Note: The proof shows that when the integrand is analytic the preceding curves $g_{1}$ and $g_{2}$ may be taken as analytic.

14. An example. Let a surface of revolution be defined by revolving an analytic open or closed curve $G$, without singularities, about an axis lying in a plane with $G_{1}$, but not intersecting $G$. Let the surface be referred to parameters $(u, v)$, of which $u$ measures the angle through which $G$ has been revolved from an initial position, and of which $v$ represents arc lengths measured along $G$ from a point chosen on $G$. The meridians $u=$ constant are all geodesics. The parallels $v=v_{0}$ are geodesics, if at the point $v=v_{0}$ the tangent to $G$ is parallel to the axis of revolution. These facts follow at once from the differential equations of the geodesics.

Let us represent the surface in the $(u, v)$ plane. Let there be given in the $(u, v)$ plane a rectangle $S$, bounded by any two curves $u=$ constant, and by any two curves $v=$ constant which represent geodesics. The rectangle $S$ can be replaced by a region $S_{1}$, interior to the rectangle, but differing from the rectangle arbitrarily little, and bounded by a curve extremal-convex relative to its interior (Lemma 2, §13). The curves $u=$ constant will form the field of extremals contemplated in $\S 8$, so that Theorem $3, \S 12$, applies to $S_{1}$.

If the curves $v=$ constant bounding the rectangle $S$ represent parallels on which the distance to the axis has a proper or improper minimum relative to neighboring parallels, then these curves $v=$ constant have no conjugate points on them, as can be readily proved. The curves $u=$ constant never 
have conjugate points on them. According to Lemma 2, $\$ 13$, the rectangle $S$ can in this case be replaced by a region $S_{1}$ slightly larger than $S$, bounded by a curve again extremal-convex relative to its interior. To $S_{1}$ Theorem $3, \S 12$, will then apply. In particular the torus presents several different types of regions $S_{1}$ to which Theorem $3, \S 12$, applies.

Part III. The type Number of A NON-Degenerate Periodic EXTREMaL

15. The function $J\left(v_{1}, \cdots, v_{n}\right)$. We start here with the same assumptions regarding the integrand $F(x, y, \dot{x}, \dot{y})$ as we made in $\S 1$. We suppose here, however, that we have given a periodic extremal $g$ of length $\omega$ and of class $C^{\prime \prime \prime}$. We again suppose $F_{1}(x, y, \dot{x}, \dot{y})>0$ along $g$.

Let $u$ be the arc length measured along $g$ in the given sense. Let $d$ be any positive constant less than the minimum distance between any two successive conjugate points on $g$. By an admissible integer $n$, and constants $u_{1}, u_{2}, \cdots$, $u_{n}$, will be meant an integer $n>1$, and constants $u_{i}$ increasing with their subscripts, all less than $u_{1}+\omega$, and such that no one of the closed intervals on $g$ consisting of points corresponding to a segment of the $u$ axis bounded by successive points of the set $u_{1}, u_{2}, \cdots, u_{n}, u_{1}+\omega$, exceeds $d$ in length. Let $h_{1}, h_{2}, \cdots, h_{n}$ be $n$ short arcs of class $C^{\prime \prime \prime}$, crossing $g$ respectively at $u_{1}$, $u_{2}, \cdots, u_{n}$, but not tangent to $g$, and let $v_{i}$ be the arc length measured along $h_{i}$ as in $\S 1$.

Let the point on $h_{i}$ at the distance $v_{i}$ from $g$ be denoted by $\left(u_{i}, v_{i}\right)$. If $v_{i}$ be sufficiently small in absolute value, the successive points of the set

$$
\left(u_{1}, v_{1}\right),\left(u_{2}, v_{2}\right), \cdots,\left(u_{n}, v_{n}\right),\left(u_{1}, v_{1}\right)
$$

can be joined by unique extremals arbitrarily near segments of $g$ between successive points on $g$ at which $u$ takes on respectively the values $u_{1}, u_{2}$, $\cdots, u_{n}, u_{1}+\omega$. The value of the integral $J$ taken along this succession of extremal segments will be again denoted by $J\left(v_{1}, \cdots, v_{n}\right)$.

16. The second partial derivatives of $J\left(v_{1}, \cdots, v_{n}\right)$. As in $\S 2, g$ can be mapped onto the $u$ axis in the $(u, v)$ plane, with the additional fact that here the transformation from the $(x, y)$ to the $(u, v)$ plane can be taken as one in which $x$ and $y$ will be functions of $u$ and $v$ with a period $\omega$ in $u$. Points in the $(u, v)$ plane whose $u$ coördinates differ by $\omega$ will be termed congruent. The periodic extremal $g$ will be represented in the $(u, v)$ plane by any segment of the $u$ axis of length $\omega$. The function $f\left(u, v, v^{\prime}\right)$ derived from $F(x, y, \dot{x}, \dot{y})$ as in $\$ 3$ will have a period $\omega$ in $u$, as will the coefficients of the J. D. E. corresponding to the extremal $v \equiv 0$. As in $\$ 2$, so here, the transformation from the $(x, y)$ to the $(u, v)$ plane may be made to preserve distances along $g$ and the $\operatorname{arcs} h_{i}$, which become respectively in the $(u, v)$. plane the curves 
$v \equiv 0$ and $u=u_{i}$. Thus the function $J\left(v_{1}, \cdots, v_{n}\right)$, set up in the preceding section, will here equal the value of the integral in the $(u, v)$ plane taken along extremal segments joining the successive points

$$
\left(u_{1}, v_{1}\right),\left(u_{2}, v_{2}\right), \cdots,\left(u_{n}, v_{n}\right),\left(u_{1}+\omega, v_{1}\right)
$$

of the $(u, v)$ plane.

$J\left(v_{1}, \cdots, v_{n}\right)$ will have a critical point when $\left(v_{1}, \cdots, v_{n}\right)=(0, \cdots, 0)$. To determine the nature of that critical point we shall examine the second partial derivatives of $J$.

In $\S 4$ the points $\left(u_{1}, v_{1}\right)$ and $\left(u_{n}, v_{n}\right)$ played a special rôle because they were adjacent to the end points of the given extremal. Here a set of formulas giving the second partial derivatives should still hold after a circular permutation of the points $\left(u_{1}, v_{1}\right), \cdots,\left(u_{n}, v_{n}\right)$. Such a circular permutation would be equivalent to advancing all the subscripts by the same integer, provided for any integer $i$ we set

$$
u_{i+n}=u_{i}+\omega, \quad v_{i+n}=v_{i} .
$$

With (2) understood we now repeat the definitions and assertions of $\$ 4$ associated with (1), (2), (3), (4), (5) and (6) of $\$ 4$.

The values of $J_{v_{i}}$ are again given by (7) of $\$ 4$ subject not to (8) of $\S 4$, but to the limitations (3) as follows:

$$
i=1,2, \cdots, n, \quad v_{0}=v_{n}, \quad v_{n+1}=v_{1} .
$$

Instead of (9) in $\S 4$ we have here

$$
J_{v_{i} j_{j}} \equiv 0, \quad i \neq j, \quad|i-j| \neq 1, \text { or } n-1,
$$

subject also to (3). Equations (10), (11), and (12), of $\S 4$, hold here exactly as given in $\S 4$. From (7) of $\S 4$, as qualified by (3) above, we obtain finally

$$
\begin{aligned}
& J_{v_{n} v_{i}}=-R\left(u_{n}\right) w_{n, n+1}^{\prime}\left(u_{n}\right), \\
& J_{v_{1} v_{n}}=R\left(u_{1}\right) w_{1,0}^{\prime}\left(u_{1}\right) .
\end{aligned}
$$

The two partial derivatives of $J$ just given in (5) were given in $\$ 4$ by (9) and were there zero; they are the only two second partial derivatives of $J$ for which the formulas of this section differ from those of $\$ 4$.

17. Periodic extremals classified: non-degenerate, simply-degenerate, and doubly-degenerate. Before going further it is necessary to consider more in detail the J. D. E. as set up in $\S 3$ for the extremal $v \equiv 0$ in the $(u, v)$ plane. We note here however that $P(u), Q(u)$, and $R(u)$, have the period $\omega$ in $u$. In terms of the solutions of this J. D. E. we distinguish three different kinds of periodic extremals $E$. 
I. There are no solutions of the J.D. E. with the period $\omega$ other than $w(u) \equiv 0$.

II. There is a set of solutions with period $\omega$ of the form $C w(u)$, where $w(u) \neq 0$, and $C$ is any constant, but no other solutions with period $\omega$.

III. Every solution of the J. D. E. has the period $\omega$.

In these three cases we shall say, respectively, that the periodic extremal is I, non-degenerate, II, simply-degenerate, and III, doubly-degenerate.

Let $p(u)$ and $q(u)$ be two solutions of the J. D. E. that satisfy the initial conditions

$$
\begin{aligned}
p(0) & =1, & q(0) & =0, \\
p^{\prime}(0) & =0, & q^{\prime}(0) & =1 .
\end{aligned}
$$

Abel's integral for these solutions becomes

$$
R(u)\left[p(u) q^{\prime}(u)-p^{\prime}(u) q(u)\right] \equiv \text { constant. }
$$

A substitution of $u=0$ and of $u=\omega$ in (2), gives the well known result

$$
p(\omega) q^{\prime}(\omega)-p^{\prime}(\omega) q(\omega)=1 .
$$

We state without proof the following known result.

.The given periodic extremal will be non-degenerate, simply-degenerate, or doubly-degenerate, according as the rank of the matrix

is 2,1 , or 0 .

$$
\left\|\begin{array}{ll}
p(\omega)-1, & q(\omega) \\
p^{\prime}(\omega) \quad, & q^{\prime}(\omega)-1
\end{array}\right\|
$$

18. Non-degenerate periodic extremal segments: convex, concave, or conjugate. Let us represent solutions $w(u)$ of the J. D. E. as curves in the $(u, w)$ plane. We will now prove the following lemma.

LEMMa 1. If the given periodic extremal is non-degenerate, a necessary and sufficient condition that in the $(u, w)$ plane every point $(0, b)$ be capable of being joined to its congruent point $(\omega, b)$ by a solution of the J. D. E., is that $u=0$ be not conjugate to $u=\omega$.

Suppose a point $(0, b)$, not $(0,0)$, can be joined to the point $(\omega, b)$ by a solution of the J. D. E. Such a solution will be of the form

$$
b p(u)+c q(u),
$$$$
b \neq 0 \text {, }
$$

where $c$ is a suitably chosen constant. Since the solution passes through $(\omega, b)$ we have

$$
b p(\omega)+c q(\omega)=b .
$$


Now if $q(\omega)$ were zero, from (2) it would follow that $p(\omega)=1$, whence the rank of the matrix (4), $\S 17$, would be less than 2 , contrary to the fact that the J. D. E. has no periodic solutions other than $w(u) \equiv 0$. Thus the condition $q(\omega) \neq 0$ is a necessary consequence of the existence of solutions of the J. D. E. joining any point $(0, b)$ to its congruent point $(\omega, b)$.

To prove that if $q(\omega) \neq 0$, any point $(0, b)$ can be joined to $(\omega, b)$ by a solution of the J. D. E., say $w(u, b)$, we exhibit such a solution, namely,

$$
w(u, b)=\frac{b}{q(\omega)}\left|\begin{array}{ll}
p(u) \quad, & q(u) \\
p(\omega)-1, & q(\omega)
\end{array}\right| .
$$

Thus the lemma is proved.

From equation (3) we obtain the following:

$$
w_{u}(\omega, b)-w_{u}(0, b)=\frac{-b}{q(\omega)}\left|\begin{array}{ll}
p(\omega)-1, & q(\omega) \\
p^{\prime}(\omega) \quad, & q^{\prime}(\omega)-1
\end{array}\right| .
$$

Now the determinant in (4) is not zero if the given periodic extremal is nondegenerate. The usual methods of the calculus of variations serve with the aid of (4) to furnish a ready proof of the following lemma.*

Lemma 2. If there be given a non-degenerate periodic extremal $g$ on which the point $u=u_{0}$ is not conjugate to $u=u_{0}+\omega$, then in the $(u, v)$ plane any point $\left(u_{0}, a\right), a \neq 0$, neighboring $\left(u_{0}, 0\right)$, can be joined to $\left(u_{0}+\omega, a\right)$ by an extremal segment $g^{\prime}$. If congruent points be regarded as identical, these extremal segments $g^{\prime}$ will make an angle $\alpha$ with themselves at $\left(u_{0}, a\right)$ measured on the side of $g^{\prime}$ towards $g$, which in magnitude will be either (Case I) always less than $\pi$, or else (Case II) always greater than $\pi$. If $u_{0}=0$, Cases I or II will occur according as the sign of

$$
M=-\frac{1}{q(\omega)}\left|\begin{array}{ll}
p(\omega)-1, & q(\omega) \\
p^{\prime}(\omega) \quad, & q^{\prime}(\omega)-1
\end{array}\right|
$$

is positive or negative.

The extremal segment $g$ taken from $u=u_{0}$ to $u=u_{0}+\omega$, will be said to be convex or concave according as $\alpha<\pi$ or $\alpha>\pi$. We shall term $M$ the test quotient.

In case a point $u=u_{0}$ on a non-degenerate periodic extremal $g$ is conjugate to its congruent point, we shall say that the segment of $g$ from $u=u_{0}$ to $u_{0}+\omega$ is a conjugate segment. It may happen in the case of a nondegenerate periodic extremal segment that every point is conjugate to its congruent point, as examples would show. In any case we see that a non-

* Compare Hadamard, Lecons sur le Calcul des Variatrons, Paris, 1910, pp. 434-435. 
degenerate periodic extremal segment from $u=u_{0}$ to $u=u_{0}+\omega$, is either convex, concave, or conjugate.

19. Conjugate points on simply-degenerate and doubly-degenerate periodic extremals. We prove the following lemma.

LEMмa 1. Let there be given a simply-degenerate, periodic extremal. Then if $w(u)$ is any one of the 1-parameter family of periodic solutions of the J.D.E. which is not identically zero, the only points which are conjugate to their congruent points are points at which $w(u)$ is zero.

Suppose the lemma false. In particular suppose that $u=a$ is a point conjugate to $u=a+\omega$, while $w(a) \neq 0$.

Let $w_{1}(u)$ be a solution of the J. D. E. which vanishes at $a$, but is not identically zero. Since $a$ is conjugate to $a+\omega$ we have

$$
w_{1}(a+\omega)=w_{1}(a)=0 .
$$

Abel's integral gives

$$
R(u)\left[w(u) w_{1}^{\prime}(u)-w^{\prime}(u) w_{1}(u)\right]=\text { constant } .
$$

Upon successively substituting $u=a$, and $u=a+\omega$ in this integral we obtain

$$
w_{1}^{\prime}(a+\omega)=w_{1}^{\prime}(a) .
$$

Because of (1) and (3) we infer that $w_{1}(u)$ is periodic. Since $w_{1}(u)$ and $w(u)$ are linearly independent, every solution of the J. D. E. is periodic, contrary to the fact that we are dealing with the simply-degenerate case, and not the doubly-degenerate case. Thus the lemma is proved.

Concerning conjugate points on doubly-degenerate periodic extremals, we say simply that every point is conjugate to its first congruent point. It would be a mistake to believe that this property is characteristic of doubly-degenerate periodic extremals, for it may occur, in particular, in the case of a nondegenerate periodic extremal, as examples would show.

20. The rank and form of the matrix of second partial derivatives of $J$. We prove the following

Theorem 4. Corresponding to the given periodic extremal $g$ of $\$ 15$, the symmetric matrix a of elements

$$
a_{i j}=J_{v_{i} v_{j}}
$$

is of rank $n, n-1$, or $n-2$, according as $g$ is non-degenerate, simply-degenerate, or doubly-degenerate. If $g$ is non-degenerate a is always in normal form.*

* Bocher, loc. cit., p. 59. 
The case where $\mathrm{g}$ is non-degenerate. To prove the theorem in this case we turn again to the $(u, w)$ plane of the J. D. E. of $\$ 18$, and in that plane consider the points

$$
\left(u_{0}, c_{0}\right),\left(u_{1}, c_{1}\right), \cdots,\left(u_{n}, c_{n}\right)
$$

of which $\left(u_{0}, c_{0}\right)$ is supposed congruent to $\left(u_{n}, c_{n}\right)$. We join the successive points of (1) by curve segments representing solutions of the J. D. E., and denote the resulting curve by $\lambda$. The curve $\lambda$ will represent a periodic solution of the J. D. E., if and only if the slope of $\lambda$ at $\left(u_{0}, c_{0}\right)$ equals its slope at $\left(u_{n}, c_{n}\right)$, and $\lambda$ has no corners at the remaining points of (1). These conditions will be fulfilled if equations (5) of $\S 5$ are satisfied for $i=1,2, \cdots$, $n$, and for $c_{0}$ and $c_{n+1}$ respectively replaced by $c_{n}$ and $c_{1}$. Let equations (5) of $\$ 5$, so altered and understood, be denoted by (5a). The J. D. E. will have a periodic solution, not identically zero, if and only if equations (5a) are satisfied by a set of constants $\left(c_{1}, \cdots, c_{n}\right)$ not all zero. Such a periodic solution is possible if, and only if, the matrix of the coefficients of $\left(c_{1}, \cdots\right.$, $c_{n}$ ) in (5a) is of rank less than $n$. With the aid of the results of $\$ 16$ this matrix is seen to be identical with the matrix a of the present theorem, provided the factor $R\left(u_{i}\right)$ be removed, for each $i$, from the $i$ th row of a. Thus the J. D. E. has no periodic solution except $w \equiv 0$, if and only if the rank of a is $n$.

That the matrix a is always arranged in normal form when $g$ is a nondegenerate periodic extremal, can now be proved after the manner of proof that the matrix a of $\$ 5$ is in normal form.

The case where $g$ is simply-degenerate. According to the proof already given the rank of a here is less than $n$. It remains to prove that the rank of $a$ is $n-1$.

Of the points $\left(u_{1}, 0\right), \cdots,\left(u_{n}, 0\right)$ at least one is not conjugate to its congruent point, for otherwise it would follow from the lemma of $\$ 19$ that the set of points $\left(u_{1}, 0\right), \cdots,\left(u_{n}, 0\right)$ are mutually conjugate, contrary to the original choice of $\left(u_{1}, u_{2}, \cdots, u_{n}\right)$. We can then suppose, without loss of generality, that $u=u_{n}$ has been so chosen as not to be conjugate to $u=u_{n}-\omega=u_{0}$.

Now the minor $A_{n-1}$, obtained from a by striking out the last row and column, is one identical in form with the minor $A_{n-1}$, considered in $\$ 5$. According to Corollary $1, \S 5, A_{n-1}$ will not be zero, since $\left(u_{0}, 0\right)$ is not conjugate to $\left(u_{n}, 0\right)$. Thus in the simply-degenerate case the rank of a is $n-1$.

The case where $g$ is doubly-degenerate. As in the preceding case the rank of $a$ is less than $n$. Further, every point on $v=0$ is here conjugate to its congruent point. In particular the point $\left(u_{0}, 0\right)$ is conjugate to its congruent point $\left(u_{n}, 0\right)$. It follows from Corollary $1, \S 5$, that $A_{n-1}=0$. But if we understand that $u_{i}+\omega=u_{i+n}$ for each integer $i$, then by advancing the subscripts 
we can bring it to pass that $\left(u_{i}, 0\right)$ is denoted by $\left(u_{n}, 0\right)$. In the matrix a the principal minor obtained by deleting the $i$ th row and column becomes the principal minor $A_{n-1}$ when $\left(u_{i}, 0\right)$ becomes $\left(u_{n}, 0\right)$. Thus all the principal $(n-1)$-rowed minors of a are zero. But according to Corollary $1, \S 5, A_{n \rightarrow 2}$ is not zero, since $\left(u_{0}, 0\right)$ is conjugate to $\left(u_{n}, 0\right)$ and cannot at the same time be conjugate to $\left(u_{n-1}, 0\right)$. Thus a is in this case of rank $n-2$, and the proof of the theorem is complete.

21. The type number of a non-degenerate periodic extremal. We prove the following

Theorem 5. If the periodic extremal $g$ of $\$ 15$ is non-degenerate, the type number $k$ of the corresponding critical point of the function $J\left(v_{1}, \cdots, v_{n}\right)$ of $\$ 15$ will be independent of the choice of $n$ among admissible integers $n$, and of the points $\left(u_{1}, \cdots, u_{n}\right)$ on $g$ among admissible points $\left(u_{1}, \cdots, u_{n}\right)$, and may be determined as follows. Setting $u_{n}-\omega=u_{0}$, let $m$ be the number of conjugate points to $u=u_{0}$, preceding $u=u_{n}$. If $u=u_{0}$ is conjugate to $u=u_{n}, k=m+1$. If $u=u_{0}$ is not conjugate to $u=u_{n}$, then $k=m$, or $m+1$, according as the segment of $g$ from $u=u_{0}$ to $u=u_{n}$ is convex or concave ( $\left.\$ 18\right)$.

The number $k$ will be called the type number of $g$.

As previously we concern ourselves here with the symmetric matrix a of which the elements are

$$
a_{i j}={ }_{a} J_{v_{i} v_{j}} .
$$

According to Theorem $4, \S 20$, the matrix a in the case of a non-degenerate periodic extremal, is of rank $n$, and arranged in normal form. The type number $k$ desired is then simply the number of changes in sign of the principal minors $A_{0}, A_{1}, \cdots, A_{n}$ (cf. $\left.\S 5, \S 6\right)$. To proceed further we agree again to set $u_{i+n}=u_{i}+\omega$, and $v_{i+n}=v_{i}$ for all integers $i$.

Now the matrix whose elements are those in $A_{n-1}$ would be identical with the matrix a of $\$ 5$ and $\S 6$, if in $\$ 5$ and $\$ 6$ we were dealing with the segment of $g$ for which $u$ lies between $u=u_{0}$ and $u=u_{n}$, and if we had chosen $n-1$ intermediate points $\left(u_{1}, \cdots, u_{n-1}\right)$ instead of $n$. To proceed further we distinguish between two cases.

Case I. The point $u=u_{0}$ is not conjugate to $u=u_{n}=u_{0}+\omega$. In this case it follows from Theorem $2, \S 6$, that the segment of $g$ for which $u$ lies between $u=u_{0}$ and $u=u_{n}$ is of type $m$, and that $A_{n-1} \neq 0$. Thus there are $m$ changes of sign in the sequence $A_{0}, A_{1}, \cdots, A_{n-1}$. Hence in Case I the number $k$ of the present theorem is $m$ or $m+1$ according to whether or not $A_{n-1}$ and $A_{n}$ have the same sign.

Now consider the $n$ equations (5) of $\$ 5$, altered as follows. We here 
replace $c_{0}$ by $c_{n}, c_{n+1}$ by $c_{1}$, and the zero of the right hand member of the $n$th equation by

$$
A_{n-1} / A_{n},
$$

while finally we here multiply the left hand member of the $i$ th one of these equations $(i=1,2, \cdots, n)$ by $R\left(u_{i}\right)$. We denote the resulting set of equations by (5b). Equations (5b) can be solved for $\left(c_{1}, \cdots, c_{n}\right)$, giving in particular for $c_{n}$ a positive value

$$
c_{n}=\frac{A_{n-1}^{2}}{A_{n}^{2}} .
$$

The interpretation of this solution is that there is a solution of the J. D.E. which in the $(u, w)$ plane passes through the points

$$
\left(u_{0}, c_{n}\right),\left(u_{1}, c_{1}\right),\left(u_{2}, c_{2}\right), \cdots,\left(u_{n}, c_{n}\right),
$$

whose slope at the point $\left(u_{n}, c_{n}\right)$ minus its slope at $\left(u_{0}, c_{n}\right)$ equals the fraction (1) divided by $R\left(u_{n}\right)$.

This difference of slopes, and hence the sign of (1), is readily seen to be positive or negative according as the segment of $g$ taken from $u=u_{0}$ to $u=u_{n}$ is convex or concave in the sense of $\S 18$. Thus in Case I, $k$ equals $m$ or $m+1$ according as the segment of $g$ from $u=u_{0}$ to $u=u_{n}$ is convex or concave.

Case II. The point $u=u_{0}$ is conjugate to $u=u_{n}=u_{0}+\omega$. In this case $A_{n-1}=0$, according to Corollary I, $\S 5$. Since $u=u_{0}$ is conjugate to $u=u_{n}$, it cannot be conjugate to $u=u_{n-1}$. If then Theorem $2, \S 6$, be applied to the segment of $g$ from $u=u_{0}$ to $u=u_{n-1}$, we find that that segment is of type $m$, since there are $m$ points conjugate to $u_{0}$ preceding $u_{n-1}$. Hence there are $m$ changes of sign in $A_{0}, A_{1}, \cdots, A_{n-2}$. But according to the theory of regularly arranged quadratic forms, when $A_{n-1}=0, A_{n-2}$ and $A_{n}$ have opposite signs. Thus there are $m+1$ changes of sign in $A_{0}, A_{1}, \cdots, A_{n}$. Hence $k=m+1$ in this case.

We can now prove that the type number $k$ is independent of the choise of $n$, and $(U)=\left(u_{1}, u_{2}, \cdots, u_{n}\right)$, among admissible integers $n$ and points $(U)$.

The formulas of $\$ 16$ and $\$ 4$ show that the partial derivatives

$$
{ }_{0} J_{v_{\boldsymbol{i}} v_{j}}
$$

are continuous functions of the position of admissible points $(U)$. Now any admissible point $(U)$ can be varied continuously through admissible points $(U)$ into any other admissible point $(U)$ for which $n$ is the same. During such a variation the terms of the sequence 


$$
A_{0}, A_{1}, \cdots, A_{n}
$$

will vary continuously, and $A_{n}$ will never be zero. We can now prove that there can be no variation in the total count of changes of sign in (2). This will certainly be true if no member of the sequence (2) is ever zero. If $A_{i}$ becomes zero (where $i$ cannot be zero or $n$ ) then $A_{i-1}$ and $A_{i+1}$ will have opposite signs at that stage of the variation. ${ }^{*}$ Thus there can be no variation in the total count of changes of sign in (2), and the statement in italics is proved, except for possible changes of $n$.

That permissible changes in $n$ do not alter the number $k$ follows from the fact that, if we hold $u_{0}$ fast and introduce or remove any set of points $u_{i}$ so as to have an admissible set $u_{i}$ left, the number $k$, as determined under Cases I and II, will be the same before and after the change. Thus the theorem is completely proved.

Part IV. The type-number of a Degenerate Periodic extremal

22. Simply-degenerate, isolated, analytic, periodic, extremals. By an isolated periodic extremal is understood a periodic extremal $g$, in the neighborhood of which there is no other periodic extremal with a length neighboring that of $g$. In this section and the two following we assume that we are dealing with a periodic extremal g given in the region $S$ of $\S 1$. Concerning $F(x, y, \dot{x}, \dot{y})$ we make the same assumptions as in $\$ 1$ together with the assumption that $F(x, y, \dot{x}, \dot{y})$ be analytic for the values of $(x, y, \dot{x}, \dot{y})$ admitted. The extremal $g$ is to be without multiple points, isolated, simply-degenerate, and analytic. Along $g, F_{1}(x, y, \dot{x}, \dot{y})$ is to be positive. We denote g's length by $\omega$.

The region neighboring $g$ can be mapped conformally on the region neighboring the $u$ axis in the $(u, v)$ plane in such a fashion that $g$ corresponds to any segment of the $u$ axis of length $\omega$, and so that congruent points $(u, v)$ and $(u+\omega, v)$ correspond to the same point $(x, y)$ in $S$, but that otherwise the transformation is one-to-one. As in $\S 3$ we can derive from $F(x, y, \dot{x}, \dot{y})$ an integrand $f\left(u, v, v^{\prime}\right)$ corresponding to which $v=0$ is an extremal. According to the lemma of $\S 19$ only a finite number of points of $g$ are conjugate to their congruent points in the simply-degenerate case. Let $(u, v)=(0,0)$ be one of the points of $g$ not congruent to its conjugate point. As is well known, for a sufficiently small constant $a \neq 0$, any point $(0, a)$ can be joined to its congruent point $(\omega, a)$ by an extremal segment $g^{\prime}$ neighboring $g$. Further, if congruent points be considered as identical these extremal segments $g^{\prime}$ make an angle with themselves at $(\omega, a)$ measured on the side of $g^{\prime}$ toward $g$ which in magnitude will now be shown to be either

* Bocher, loc. cit., p. 147. 
(I) Less than $\pi$ for all vertices $(\omega, a)$ on one side of $g$, and greater than $\pi$ for $(\omega, a)$ on the other side of $g$;

(II) Less thain $\pi$ for all vertices $(\omega, a)$ on either side of $g$; or

(III) Greater than $\pi$ for all vertices $(\omega, a)$ on either side of $g$.

Further, whether I, II, or III occurs depends only upon $g$ and the choice of the point $(u, v)=(0,0)$.

The family of extremals passing through the point $(u, v)=(0, a)$ with a slope $b$ can be represented in the form

$$
v=A(u, a, b), \quad a^{2}+b^{2} \leqq e,
$$

where $A(u, a, b)$ is a real analytic function of $(u, a, b)$ for $u$ on any finite segment of the $u$ axis and for a corresponding positive constant $e$ sufficiently small, where further

$$
\begin{aligned}
a & \equiv A(0, a, b), \\
b & \equiv A_{u}(0, a, b) .
\end{aligned}
$$

The condition that an extremal $(a, b)$ pass from a point $(0, a)$ to $(\omega, a)$ is that

$$
A(\omega, a, b)-a=0
$$

Now we have

$$
A_{b}(\omega, 0,0) \neq 0,
$$

since $(0,0)$ has been chosen so as not to be conjugate to $(\omega, 0)$. Hence $(4)$ possesses a solution

$$
b=\beta(a), \quad \beta(0)=0,
$$

where $\beta(a)$ is analytic in $a$ at $a=0$. The slope of an extremal $(a, b)$ given by (6) at $(\omega, a)$, minus its slope at $(0, a)$, is

$$
A_{u}(\omega, a, \beta(a))-\beta(a) \text {. }
$$

This function is not identically zero in $a$. For otherwise extremals $(a, b)$ given by (6) would make up a continuous family of periodic extremals, a case which has been barred. Hence we can write

$$
A_{u}(\omega, a, \beta(a))-\beta(a)=\bar{A}(a)=a^{r} k(a), \quad k(0) \neq 0, r>1,
$$

where $k(a)$ is analytic in $a$ at $a=0$. That $r$ is an integer exceeding one is seen from the fact that

$$
\bar{A}^{\prime}(0)=-\frac{1}{A_{b}(\omega, 0,0)}\left|\begin{array}{ll}
A_{a}(\omega, 0,0)-1, & A_{b}(\omega, 0,0) \\
A_{u, a}(\omega, 0,0), & A_{u, b}(\omega, 0,0)-1
\end{array}\right| .
$$


In terms of $p(u)$ and $q(u)$ of $\$ 17$ this gives

$$
\bar{A}^{\prime}(0)=-\frac{1}{q(\omega)}\left|\begin{array}{ll}
p(\omega)-1, & q(\omega) \\
p^{\prime}(\omega) \quad, & q^{\prime}(\omega)-1
\end{array}\right| .
$$

Now the determinant in (10) is always zero if the periodic extremal is degenerate $(\$ 17)$. Thus here $\bar{A}^{\prime}(0)=0$ and $r>0$. The statements in italics follow at once from (8).

23. A simply-degenerate periodic extremal. Cases: concave-convex, concave, convex. A simply-degenerate, isolated, analytic, periodic, extremal $g$, taken from $u=0$ to $u=\omega$ will be said to be concave-convex, convex, or concave, according as I, II, or III of the preceding section holds. We have previously given similar definitions for a non-degenerate periodic extremal (\$18) involving the terms convex and concave. The concave-convex case did not present itself in the non-degenerate case. In the present case we state in terms of $r$ and $k(0)$ in (8) of the preceding section that the segment of $g$ from $u=0$ to $u=\omega$ is

(I) Concave-convex when $r$ is even,

(II) Convex when $r$ is odd and $k(0)>0$,

(III) Concave when $r$ is odd and $k(0)<0$.

24. The type number of a simply-degenerate periodic extremal. To define the type number of $g$ we are going to modify the integrand $f\left(u, v, v^{\prime}\right)$ in such a fashion that we shall no longer have to do with a degenerate periodic extremal. Let $h(u)$ be any real analytic function of $u$ with a period $\omega$, and $\mu$ be a parameter. An integrand of the form

$$
f\left(u, v, v^{\prime}\right)+\mu v h(u)
$$

reduces for $\mu=0$ to the original integrand. We will now prove the following theorem.

TheOREM 6. Concerning the simply-degenerate periodic extremal $g$ of $\$ 22$, on which $(0,0)$ can and will be chosen so as not to be conjugate to $(\omega, 0)$, we can say the following. It is possible to choose a function $h(u)$ real and analytic in $u$ with a period $\omega$, and values of $\mu$ arbitrarily near $\mu=0$ in such a fashion, that if $f\left(u, v, v^{\prime}\right)$ be replaced by the integrand (1) then we have the following:

(A) If $g$, taken from $u=0$ to $u=\omega$, is convex-concave, the modified problem will possess no extremals neighboring $g$ of period $\omega$ in $u$.

( $\left.\mathrm{B}_{1}\right)$ If $g$, taken from $u=0$ to $u=\omega$, is convex or concave, the modified problem will possess just one extremal $E$ neighboring $g$ of period $\omega$ in $u$. 
$\left(\mathrm{B}_{2}\right)$ The extremal $E$ of $\left(\mathrm{B}_{1}\right)$ will be non-degenerate, and if there are on $g$ just $m$ points conjugate to $u=0$ and preceding $u=\omega$, the type number of $E$ as determined in Theorem $5, \S 21$, will be $m$ or $m+1$ according as $g$, taken from $u=0$ to $u=\omega$, is convex or concave.

The choice of $h(u)$. The extremals which correspond to the integrand (1) for values of $\mu$ neighboring $\mu=0$, and which lie in the neighborhood of the extremal $\mu=0$, can be represented in the form

$$
v=B(u, a, b, \mu), \quad a^{2}+b^{2}+\mu^{2} \leqq e,
$$

with

$$
\begin{aligned}
& a \equiv B(0, a, b, \mu), \\
& b \equiv B_{u}(0, a, b, \mu),
\end{aligned}
$$

where $B(u, a, b, \mu)$ is analytic in its arguments, for $u$ on any closed interval, and $e$ a correspondingly sufficiently small positive constant.

We will show that we can choose $h(u)$ so that

$$
\begin{aligned}
B_{\mu}(\omega, 0,0,0) & \neq 0, \\
B_{u \mu}(\omega, 0,0,0) & \neq 0 .
\end{aligned}
$$

Differentiation of the Euler equation with respect to $\mu$ will show that the function $B_{\mu}(u, 0,0,0)$, set equal to $w(u)$, satisfies

$$
R w^{\prime \prime}+R^{\prime} w^{\prime}+\left(Q^{\prime}-P\right) w=h(u)
$$

where $R(u), P(u)$, and $Q(u)$ are the functions already used in the J. D. E. set up for the extremal $v \equiv 0$, when $\mu=0$.

It follows from (3) and (4) that we have initially

$$
\begin{aligned}
w(0) & =B_{\mu}(0,0,0,0)=0, \\
w^{\prime}(0) & =B_{u \mu}(0,0,0,0)=0 .
\end{aligned}
$$

If we make use of the functions $p(u)$ and $q(u)$ of $\$ 17$, it is readily seen that this solution $w(u)$ of $(7)$ is given by

In particular

$$
w(u)=B_{\mu}(u, 0,0,0)=\int_{0}^{u} \frac{h(t)}{R(0)}\left|\begin{array}{ll}
p(t), & q(t) \\
p(u), & q(u)
\end{array}\right| d t .
$$

$$
\begin{gathered}
B_{\mu}(\omega, 0,0,0)=\int_{0}^{\omega} \frac{h(t)}{R(0)}\left|\begin{array}{ll}
p(t), & q(t) \\
p(\omega), & q(\omega)
\end{array}\right| d t, \\
B_{\mu u}(\omega, 0,0,0)=\int_{0}^{\omega} \frac{h(t)}{R(0)}\left|\begin{array}{ll}
p(t), & q(t) \\
p^{\prime}(\omega), & q^{\prime}(\omega)
\end{array}\right| d t .
\end{gathered}
$$


Now the coefficients of $h(t)$ in the integrands of (9) and (10) are respectively 0 and $R_{(0)}^{-1}$ for $t=\omega$. They are both positive for $t$ in an interval

$$
\omega-e<t<\omega,
$$

if $e$ be a sufficiently small positive constant. Let $h_{1}(u)$ be a function which is identically zero as $t$ ranges from $t=0$ to $t=\omega$, except that in the interval (11), $v=h_{1}(u)$ shall be equal to the $v$ ordinate on a semicircle with end points at $(\omega-e, 0)$ and $(\omega, 0)$ and on which $v>0$. If in (9) and (10), $h(t)$ be replaced by $h_{1}(t)$, the resulting integrals would both be positive. Another fact of importance is that if $e$ in (11) be a sufficiently small positive constant the corresponding function $h_{1}(t)$ will not only make the integrals (9) and (10) positive but will cause the ratio of the integral (9) to the integral (10) to be arbitrarily small. Now $h_{1}(t)$ can be approximated by a number of terms of a Fourier series with period $\omega$. The resulting function, which will serve as our definition of $h(t)$, can be taken as a function approximating $h_{1}(t)$ so closely that for this choice of $h(t)$ in (9) and (10) the ratio of $B_{\mu}(\omega, 0,0,0)$ to $B_{u \mu}(\omega, 0,0,0)$ will be arbitrarily small, and both $B_{\mu}(\omega, 0,0,0)$ and $B_{u \mu}(\omega, 0$, $0,0)$ will be positive.

We can now settle the question of the existence of periodic extremals near the extremal $v \equiv 0$ corresponding to the integrand (1) when $\mu$ is near $\mu=0$. The conditions for a periodic extremal neighboring $v \equiv 0$ for $\mu$ near $\mu=0$ are

$$
\begin{aligned}
B(\omega, a, b, \mu)-a & =0, \\
B_{u}(\omega, a, b, \mu)-b & =0 .
\end{aligned}
$$

Now equation (12) reduces for $\mu=0$ to equation (4) of $\$ 22$. But (4) of $\$ 22$ is satisfied by the function $b=\beta(a)$ of (6), $\$ 22$. Hence (12) is satisfied by $b=\beta(a)$ and $\mu=0$. Further

$$
B_{b}(\omega, 0,0,0) \neq 0,
$$

since on the extremal $v \equiv 0$, the point $(0,0)$ was chosen not conjugate to $(\omega, 0)$. Hence (12) admits a solution of the form

$$
b=b(a, \mu)=\beta(a)+\mu R(a, \mu),
$$

where $R(a, \mu)$ is analytic in $a$ and $\mu$, at $a=0$ and $\mu=0$.

We are next concerned with solving (13) subject to (12), that is with solving

$$
B_{u}[\omega, a, b(a, \mu), \mu]-b(a, \mu)=0 .
$$

Using the fact that $b(a, 0)=\beta(a)$ we obtain the identity

$$
B_{u}[\omega, a, b(a, 0), 0]-b(a, 0) \equiv A_{u}[\omega, a, \beta(a)]-\beta(a),
$$


which becomes with the aid of (8), $\$ 22$,

$$
\equiv a^{r} k(a), \quad k(0) \neq 0 .
$$

Hence (16) becomes

$$
B_{u}[\omega, a, b(\alpha, \mu), \mu]-b(a, \mu)=a^{r} k(a)+\mu S(a, \mu)=0,
$$

where $S(a, \mu)$ is analytic at $a=0$ and $\mu=0$. We can solve (17) for $\mu$ if $S(0,0) \neq 0$. But $S(0,0)$ is the partial derivative with respect to $\mu$, at $(a, \mu)$ $=(0,0)$, of the left hand member of $(17)$. Thus

$$
S(0,0)=\left[1-B_{u b}(\omega, 0,0,0)\right] \frac{B_{\mu}(\omega, 0 ; 0,0)}{B_{b}(\omega, 0,0,0)}+B_{\mu u}(\omega, 0,0,0) .
$$

Now as we have seen we can choose $h(t)$ so that $B_{\mu u}(\omega, 0,0,0)$ will be positive at the same time that the ratio of $B_{\mu}(\omega, 0,0,0)$ to $B_{\mu u}(\omega, 0,0,0)$ is arbitrarily small, so that the term $B_{\mu u}(\omega, 0,0,0)$ will dominate the sign in (18). Thus $S(0,0) \neq 0$ for a proper choice of $h(t)$. Hence, (17) admits a solution of the form

$$
\mu=\mu(a)=a^{r} d(a), \quad d(0) \neq 0,
$$

where $d(a)$ is analytic at $a=0$. The solution of (12) and (13) is now given by (19), taken with (15).

Final proof of (A). Now the integer $r$ in (19) is the integer $r$ in (8), \$22. If the given periodic extremal is convex-concave, $r$ is even. In this case let $\mu$ be chosen arbitrarily small, different from zero, and opposite in sign to $d(0)$ in (19). For this $\mu,(19)$ will admit no real solution $a$ neighboring $a=0$, and corresponding to this $\mu$ the problem will possess no periodic extremal neighboring the extremal $v \equiv 0$.

Proof of $\left(B_{1}\right)$. If the given periodic extremal is convex or concave, $r$ is odd, and corresponding to any value of $\mu$ say $\mu_{1} \neq 0$, neighboring $\mu=0$, (19) gives a value of $a$, say $a_{1}$, and (15) then a value of $b$, say $b_{1}$, corresponding to which the modified problem for which $\mu=\mu_{1}$ will possess a single periodic extremal $E$, with initial point $\left(0, a_{1}\right)$ and initial slope $b_{1}$.

Proof of $\left(\mathrm{B}_{2}\right)$. The sign of the test quotient $M$ of $(5), \S 18$, determined for $E$. To show that $E$ is non-degenerate it will be sufficient to prove that the test quotient $M$ of Lemma $2, \S 18$, evaluated for $E$, is not zero. According to the results of $\$ 18, E$, taken from $u=0$ to $u=\omega$, will be convex or concave according as $M$ is positive or negative. By thus determining the sign of $M$ we intend to prove that the non-degenerate extremal $E$ is convex or concave according as the given simply-degenerate extremal $v \equiv 0$ is convex or concave, taking both extremals from $u=0$ to $u=\omega$. 
More generally the quotient $M$ of $\$ 18$ set up for any extremal with parameters $(a, b, \mu)$ in (2), will be denoted by $M(a, b, \mu)$. We have

$$
\frac{-1}{B_{b}}\left|\begin{array}{ll}
B_{a}-1, & B_{b} \\
B_{u a}, & B_{u b}-1
\end{array}\right|=M(a, b, \mu),
$$

where in each of the partial derivatives we set $u=\omega$, but where $(a, b, \mu)$ may take on any values neighboring $(0,0,0)$. The form (20) shows that in terms of the function $b(a, \mu)$ of (15)

$$
M[a, b(a, \mu), \mu] \equiv \frac{\partial}{\partial a}\left[B_{u}\{\omega, a, b(a, \mu), \mu\}-b(a, \mu)\right] .
$$

With the aid of (17) we therefore have

$$
M[a, b(a, \mu), \mu] \equiv r a^{r-1} k(a)+a^{r} k^{\prime}(a)+\mu S_{a}(a, \mu) .
$$

We shall evaluate (21) for values of $\mu$ given by (19), thereby evaluating $M(a, b, \mu)$ for values of $(a, b, \mu)$ which correspond to periodic extremals. Thus

$$
M[a, b\{a, \mu(a)\}, \mu(a)] \equiv r a^{r-1} k(0)+\cdots, \quad k(0) \neq 0,
$$

where the terms omitted are of higher order than $r-1$ in $a$.

Now according to the results of $\$ 23$ the given simply-degenerate extremal $v \equiv 0$, taken from $u=0$ to $u=\omega$, is convex or concave when $r$ is odd, and more particularly is convex or concave according as $k(0)$ is positive or negative. It follows from (22) and (19) that if $E$ corresponds to a parameter $\mu \neq 0$, sufficiently small in absolute value, then $E$ will be convex or concave according as the extremal $v \equiv 0$ is convex or concave ( $\$ 18)$.

Finally the type of the non-degenerate extremal $E$, determined according to Theorem 5, $\$ 21$, equals the number $m$ of points on $E$ conjugate to $u=0$ and preceding $u=\omega$, plus one when $E$ is concave, and exactly $m$ when $E$ is convex. But this number $m$ will equal the corresponding number determined on the extremal $v=0$, provided only that $\mu$ be sufficiently small in absolute value. Thus part $\left(B_{2}\right)$ of the theorem is proved.

In accordance with the results of the preceding theorem, the given simplydegenerate periodic extremal $g$, in case it is convex or concave, will be said to be equivalent in type to the non-degenerate extremal $E$ whose existence is affirmed by the preceding theorem, and in case it is convex-concave will be said to be equivalent to a null set of extremals and to be neutral in type.

25. A doubly-degenerate, isolated, analytic, periodic, extremal. We make the same assumptions here as we made in the case of a simplydegenerate periodic extremal (\$22), except that here the given extremal $g$ 
is to be doubly-degenerate $(\S 17)$. We will reduce the determination of the type of $g$ to the cases already considered, that is, the non-degenerate and simply-degenerate cases.

ThEOREM 7. Concerning the preceding doubly-degenerate, isolated, periodic extremal $g$, and the corresponding simplified integrand $f\left(u, v, v^{\prime}\right)$ for which $g$ becomes the extremal $v \equiv 0$, we can say the following. It is possible to choose a function $h(u)$ analytic and periodic in $u$ with the period $\omega$, such that the extremals corresponding to the modified integrand

$$
f\left(u, v, v^{\prime}\right)+\mu v h(u),
$$

for properly chosen values of the parameter $\mu$, neighboring $\mu=0$, will include at most a finite set $\sigma$ of extremals with the period $\omega$ in $u$ that lie in the neighborhood of $v \equiv 0$, and such that none of these extremals will be doubly-degenerate.

To prove Theorem 7 we proceed as in the proof of Theorem 6, $\$ 24$. We represent the extremals neighboring $v \equiv 0$ as in $\$ 24$ and choose $h(u)$ in the same way, so that we may regard equations (1) to (13) of $\$ 24$ as holding here. At this point the two proofs diverge, since (14) of $\$ 24$ does not hold here. In terms of the function $A(u, a, b)$ of $\$ 22$ of the unmodified problem, equations (12) and (13) of $\$ 24$ become

$$
\begin{aligned}
& B(\omega, a, b, \mu)-a \equiv A(\omega, a, b)-a+\mu D(a, b, \mu)=0, \\
& B_{u}(\omega, a, b, \mu)-b \equiv A_{u}(\omega, a, b)-b+\mu E(a, b, \mu)=0,
\end{aligned}
$$

where $D(a, b, \mu)$ and $E(a, b, \mu)$ are analytic in $(a, b, \mu)$ at $(a, b, \mu)=(0,0,0)$. With the aid of (5) and (6), §24, we see that

$$
\begin{aligned}
& D(0,0,0)=B_{\mu}(\omega, 0,0,0) \neq 0, \\
& E(0,0,0)=B_{u \mu}(\omega, 0,0,0) \neq 0 .
\end{aligned}
$$

Because of (3) and (4), (1) and (2) can be solved for $\mu$. These solutions take the forms, respectively,

$$
\begin{aligned}
\mu & =[A(\omega, a, b)-a] G(a, b), \\
\mu & =\left[A_{u}(\omega, a, b)-b\right] H(a, b),
\end{aligned}
$$

where $G(a, b)$ and $H(a, b)$ are analytic at $(a, b)=(0,0)$ and do not vanish there. To solve (5) and (6) simultaneously we are led to the equation

$$
[A(\omega, a, b)-a] G(a, b)=\left[A_{u}(\omega, a, b)-b\right] H(a, b) .
$$

We distinguish between two cases:

Case I: Equation (7) holds identically.

Case II: Equation (7) does not hold identically. 
The proof in Case I. In this case neither of the differences

$$
A(\omega, a, b)-a, \quad A_{u}(\omega, a, b)-b
$$

can vanish along any real analytic arcs in the $(a, b)$ plane neighboring $(a, b)$ $=(0,0)$. For if either of these two differences vanished along such real arcs, according to (7) the other difference would also so vanish, and the extremals for the unmodified problem $\mu=0$ would include a family of periodic extremals contrary to the assumption that $g$ is isolated. Except at $(0,0)$, the two members of (7) must then be of one sign, say positive, throughout the neighborhood of $(a, b)=(0,0)$. If then $\mu$ be chosen negative and sufficiently near $\mu=0$, there will be no solution of (5) and (6) and hence no solution of (11) and (12). For each such choice of $\mu$ the modified problem will possess no periodic extremals neighboring $g$.

Proof in Case II. It may happen in this case that (7) possesses no real solutions neighboring $(a, b)=(0,0)$ other than $(0,0)$. If this occurs, then for any choice of $\mu \neq 0$, sufficiently near $\mu=0,(1)$ and (2) admit no real solutions $(a, b, \mu)$ and the modified problem possesses no periodic extremals neighboring $g$.

If, on the other hand, (7) possesses real solutions $(a, b) \neq(0,0)$, arbitrarily near $(a, b)=(0,0)$, these real solutions will make up a finite number of real analytic arcs representable in a one-to-one manner by function pairs of the form

$$
\begin{array}{ll}
a=a(t), & a(0)=0, \\
b=b(t), & b(0)=0,
\end{array}
$$

where $a(t)$ and $b(t)$ are real analytic functions of $t$ at $t=0$, and are not both identically zero. The differences

$$
\begin{aligned}
& A[\omega, a(t), b(t)]-a(t), \\
& A_{u}[\omega, a(t), b(t)]-b(t)
\end{aligned}
$$

cannot both be identically zero, for otherwise $a(t), b(t)$ would correspond in the unmodified problem to a continuous family of periodic extremals. On the other hand neither of the functions in (9) can be identically zero in $t$ without the other being identically zero in $t$, as follows from (7). We can then set

$$
A[\omega, a(t), b(t)]-a(t) \equiv t^{\bullet} F(t), \quad F(0) \neq 0, \quad s>0,
$$

where $F(t)$ is analytic at $t=0$. From (5) it follows that the values of $\mu$ that go with (8) to give a solution of (1) and (2) are representable in the form

$$
\mu=\mu(t) \equiv t^{\bullet} K(t), \quad K(0) \neq 0,
$$


where $K(t)$ is analytic at $t=0$. For any particular $t$, neighboring $t=0$; and corresponding values of $a(t), b(t)$, and $\mu(t)$, the corresponding extremal $g^{\prime}$ will be periodic.

Among the conditions that $g^{\prime}$ be doubly-degenerate are that for the value of $t$ that gives $g^{\prime}(\S 17)$,

$$
\begin{aligned}
& B_{a}[\omega, a(t), b(t), \mu(t)]=1, \\
& B_{b}[\omega, a(t), b(t), \mu(t)]=0 .
\end{aligned}
$$

We proceed to show that (12) and (13) do not hold identically in $t$. For if (12) and (13) did hold identically in $t$, a performance of the following indicated differentiation would show that

$$
\frac{\partial}{\partial t}\{B[\omega, a(t), b(t), \mu]-a(t)\} \equiv 0, \quad \mu=\mu(t),
$$

was an identity in $t$, where, as indicated, $\mu$ is to be held fast during the differentiation and set equal to $\mu(i)$ thereafter. If use be made of (1) and (10) we obtain the identity

$$
B[\omega, a(t), b(t), \mu]-a(t) \equiv t^{\circ} F(t)+\mu D[a(t), b(t), \mu],
$$

and the partial derivative (14) becomes, upon using (11) and (15),

$$
s t^{t^{-1}} F(t)+t^{\bullet} F^{\prime}(t)+t^{t} K(t)\left[D_{a} \cdot a^{\prime}(t)+D_{b} \cdot b^{\prime}(t)\right] .
$$

This function does not vanish identically since $s>0$ and $F(0) \neq 0$. Hence (12) and (13) do not hold identically, and accordingly hold simultaneously for no value of $t$, neighboring $t=0$, other than $t=0$.

Now to any real value of $\mu$ not zero, but sufficiently near zero, there will correspond, under (11), either no real value, one real value, or two real values of $t$, according to the evenness, or oddness of $s$, and the sign of $K(0)$. To such a $\mu$ there will then correspond, by virtue of (8), no periodic extremal, one periodic extremal, or two periodic extremals, and none of these extremals will be doubly-degenerate, since (12) and (13) will not both hold.

Similarly there may arise a finite number of other periodic extremals from real solutions of (7) other than $a(t), b(t)$, but in any case for a $\mu \neq 0$, and sufficiently near $\mu=0$, these periodic extremals will not be doublydegenerate. Thus the theorem is proved.

Let the set $\sigma$ of periodic extremals appearing in Theorem 7 be modified by replacing each simply-degenerate periodic extremal $g_{1}$ of $\sigma$ by an equivalent non-degenerate extremal, or a null set of extremals, according to the conventions at the end of \$24. The set $\sigma_{1}$ of non-degenerate extremals thereby obtained will be 
said to be equivalent in type to the given doubly-degenerate extremal $g$. If neither $\sigma$ nor $\sigma_{1}$ contains any extremals, $g$ will be said to be equivalent to a null set of extremals, and to be of neutral type.

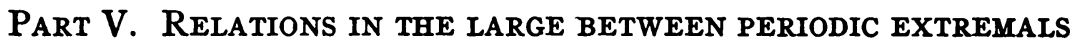

26. The integrand and regions $S$ and $S_{1}$. We make here again the assumptions I and II of $\S 7$ and $\S 8$, qualifying $F$ and $S$. We replace III by III', and IV by IV' as follows:

III'. Let there be given a closed region $S_{1}$ consisting of points interior to $S$ bounded by two simple closed curves $\beta_{1}$ and $\beta_{2}$, of which $\beta_{2}$ lies within $\beta_{1}$. We suppose both $\beta_{1}$ and $\beta_{2}$ consist of a finite number of analytic arcs without singularities and are extremal-convex relative to $S_{1}$ in the sense of III $\$ 8$.

IV'. We assume that we have in $S$ a proper field of extremals representable in the form

$$
x=h(u, v), \quad y=k(u, v),
$$

where $u$ is the parameter and $v$ is the arc length measured along the extremals, and $h(u, v)$ and $k(u, v)$ have a period $\omega$ in $u$. We assume further that for any constant $a$ and interval

$$
a \leqq u<a+\omega,
$$

the field (1) covers $S_{1}$ in a one-to-one manner, and that at each point $(u, v)$ that corresponds to a point $(x, y)$ in $S_{1}$ the functions (1) are single-valued and analytic in $u$ and $v$, and

$$
\left|\begin{array}{ll}
h_{u}, & h_{v} \\
k_{u}, & k_{v}
\end{array}\right| \neq 0 .
$$

The lemma of $\$ 8$ will here be replaced by the following lemma of which the method of proof is very similar to that used in $\$ 8$. No proof need be given.

Lemмa 1. The region $S_{1}$ of the $(x, y)$ plane will correspond under (1) to a region $R$ of the $(u, v)$ plane bounded by two unending arcs of the form

$$
\begin{array}{ll}
v=A(u), & A(u+\omega)=A(u), \\
v=B(u), & B(u+\omega)=B(u), \quad A(u)<B(u),
\end{array}
$$

where $A(u)$ and $B(u)$ are of class $C$ for all values of $u$, and analytic in $u$ except for a finite set of values of $u$, on any interval of the form (2). The interior points of $R$ are the points $(u, v)$ such that

$$
A(u)<v<B(u) \text {. }
$$

The correspondence between $S_{1}$ and any set of points of $R$ limited as in (2) will be one-to-one. 
As in $\S 9$, so here, it follows that all extremals, except the extremals $u=$ constant, are representable in the form

$$
v=M(u),
$$

where $M(u)$ is an analytic function of $u$ for all values of $u$ that give points $(u, v)$ in $R$. Further any extremal joining two points in $R$ will have no points on the boundary of $R$, with the possible exception of its end points.

We shall concern ourselves at first with periodic extremals continuously deformable in $S_{1}$, in the $(x, y)$ plane, into either one of the two closed boundary curves of $S_{1}$, taken just once. In $R$, in the $(u, v)$ plane, these extremals will be representable in the form (5), with $M(u)$ possessing the period $\omega$. A point of difference between the developments for periodic extremals and those for extremals joining two fixed points $A$ and $B$, is that in the latter case we were able to prove, under hypotheses I, II, III, and IV, of $\$ 7$ and $\S 8$, that there were at most a finite number of extremals joining $A$ to $B$, while in the case of periodic extremals the hypotheses I, II, III', and IV $^{\prime}$ are not sufficient to bar the existence of analytic families of periodic extremals lying in $S_{1}$ and deformable into a boundary of $S_{1}$. Simple examples can be given to show the truth of this statement. However, cases where families of periodic extremals exist are certainly specialized in that the differential equation of the first variation, that is the J. D. E., set up for each member of such families, must possess a periodic solution not identically zero. Not only is this true but the differential equations of the variations of orders higher than the first must all possess periodic solutions. In excluding such families we are therefore excluding exceptional cases. We are the more justified in such exclusion by the fact that we are developing a theory that will serve to prove the existence of additional periodic extremals when a finite set of such extremals is given. If infinite sets of mutually deformable periodic extremals existed, then more than we hoped to prove would be granted. We state here the fundamental lemma which replaces Lemma 2 of $\$ 11$.

LEMma 2. Let there be given regions $S$ and $S_{1}$ satisfying I and II of \$7, and $\mathrm{III}^{\prime}$ and $\mathrm{IV}^{\prime}$ of this section. In $S_{1}$ suppose there are at most a finite number of periodic extremals continuously deformable into either boundary of $S_{1}$, taken just once, and that all of these extremals are non-degenerate (\$17). Let the number of these extremals which are of type $k(\$ 21)$ be denoted by $M_{k}$. Let $m$ be the maximum of the integers $k$. Then between these numbers $M_{k}$ the relations (R) of $\$ 11$ hold. 
This lemma is proved with the aid of the fundamental lemma on critical points, $\S 11$, and Theorem $5, \S 21$, in a manner similar to the manner of proof of Lemma 2 , §11.

27. The theorem in the large. In order to remove the restriction from the above lemma that the periodic extremals appearing there be nondegenerate we cannot rely on the envelope theory as in the case of extremals joining two fixed points. The following lemma will serve our purpose. In this lemma let the $(n-1)$-dimensional hypersphere with radius $r$ and center $(A)$ in the space of $\left(v_{1}, \cdots, v_{n}\right)$ be denoted by $S^{\Lambda_{1}, \text {. }}$.

Lemma 1. Let there be given a function $J\left(v_{1}, \cdots, v_{n}\right)$ of class $C^{\prime \prime \prime}$ at each point $(V)=\left(v_{1}, \cdots, v_{n}\right)$ of an open n-dimensional region $\Sigma$. Let $(A)$ $=\left(a_{1}, \cdots, a_{n}\right)$ be a point of $\Sigma$ at which $J\left(v_{1}, \cdots, v_{n}\right)$ has an isolated critical point. Let $J\left(v_{1}, \cdots, v_{n}, \mu\right)$ be a function of class $C^{\prime \prime \prime}$ for $(V)$ in $\Sigma$, and $\mu$ in the neighborhood of $\mu=0$, and such that in $\Sigma$

$$
J\left(v_{1}, \cdots, v_{n}\right) \equiv J\left(v_{1}, \cdots, v_{n}, 0\right) .
$$

Let e be a positive constant so small that $J\left(v_{1}, \cdots, v_{n}\right)$ has no critical point other than $(A)$ within or on $S^{1,2 e}$. Then for any fixed value of $\mu \neq 0$ and sufficiently small in absolute value, it is possible to replace $J\left(v_{1}, \cdots, v_{n}\right)$ by a function $\phi\left(v_{1}, \cdots, v_{n}\right)$ of class $C^{\prime \prime \prime}$ throughout $\Sigma$, and of such sort that in $S^{1, \bullet}$

$$
\phi\left(v_{1}, \cdots, v_{n}\right) \equiv J\left(v_{1}, \cdots, v_{n}, \mu\right),
$$

while in $\Sigma$ but outside of $S^{\Lambda, 2 e}$

$$
\phi\left(v_{1}, \cdots, v_{n}\right) \equiv J\left(v_{1}, \cdots, v_{n}\right),
$$

and finally in the closed domain between $S^{1,26}$ and $S^{1,0}, \phi\left(v_{1}, \cdots, v_{n}\right)$ has no critical points.

To prove this lemma we are going to use a function $h(x)$ that is of class $C^{\prime \prime \prime}$ for all values of $x$, and is such that for the preceding constant $e$

$$
\begin{array}{ll}
h(x) \equiv 1, & |x| \leqq e, \\
h(x) \equiv 0, & |x| \geqq 2 e .
\end{array}
$$

Such a function as $h(x)$ can readily be set up in terms of the elementary functions.

For points $\left(v_{1}, \cdots, v_{n}\right)$ of the closed domain between $S^{1,28}$ and $S^{1, \bullet}$ set 


$$
D\left(v_{1}, \cdots, v_{n}, \mu\right) \equiv J\left(v_{1}, \cdots, v_{n}, \mu\right)-J\left(v_{1}, \cdots, v_{n}\right),
$$

and now define $\phi$ for the same points $\left(v_{1}, \cdots, v_{n}\right)$ as follows:

(6) $\phi\left(v_{1}, \cdots, v_{n}\right) \equiv J\left(v_{1}, \cdots, v_{n}\right)+D\left(v_{1}, \cdots, v_{n}, \mu\right) h\left(\left[v_{1}{ }^{2}+\cdots+v_{n}{ }^{2}\right]^{1 / 2}\right)$.

Now the constant $\mu$ can be chosen so small in absolute value that the function $D$ of (6), as well as each of the partial derivatives $D_{v_{i}}$, is less in absolute value than a preassigned positive constant $d$ throughout the whole region between $S^{\boldsymbol{\Lambda}, 2 e}$ and $S^{\boldsymbol{A}, \boldsymbol{e}}$. For the same domain the sum of the squares of the first partial derivatives of $J\left(v_{1}, \cdots, v_{n}\right)$ exceeds some positive constant. It follows readily from (6), that for a $\mu \neq 0$, sufficiently small in absolute value, the function $\phi$ of (6) will have no critical point between $S^{\mathbf{4}, 26}$ and $S^{1,8}$. We accordingly understand such a value of $\mu$ chosen, and hereafter held fast.

If now the function $\phi\left(v_{1}, \cdots, v_{n}\right)$ be defined at the remaining points of $\Sigma$ by (2) and (3), it is readily seen that $\phi\left(v_{1}, \cdots, v_{n}\right)$ has the properties affirmed in the lemma.

We are now in a position to state the following theorem.

THEOREM 8. Let there be given regions $S$ and $S_{1}$ and integrand $F(x, y, \dot{x}, \dot{y})$ satisfying I, II, $\mathrm{II}^{\prime}, \mathrm{IV}^{\prime}$ of $\S 7$ and $\S 26$. In $S_{1}$ suppose there are at most a finite set $T$, of periodic extremals $g$, continuously deformable into a boundary curve of $S_{1}$. In the set $T$ let each degenerate extremal be replaced by an equivalent set of non-degenerate extremals in accordance with the conventions at the ends of $\$ 24$ and $\$ 25$. Let $M_{k}$ be the number of periodic extremals of type $k$ in the resulting set, and $m$ be the maximum of the numbers $k$. Between the numbers $M_{k}$ the relations $(\mathrm{R})$ of $\$ 11$ hold.

If the above periodic extremals $g$ are all non-degenerate, the present theorem is identical with Lemma $2, \S 26$.

Suppose on the other hand that not all of the above periodic extremals $g$ are non-degenerate. To be specific, suppose the above set includes a simply-degenerate periodic extremal $g_{1}$ which taken from $u=0$ to $u=\omega$ is convex or concave. Then according to Theorem 6 of $\$ 24$ it will be possible to modify the integrand by the introduction of a parameter $\mu$ in such a fashion, that for a suitably chosen value of $\mu$, say $\mu_{1}$, arbitrarily small in absolute value, there will appear, instead of $g_{1}$, in the neighborhood of $g_{1}$, a non-degenerate extremal $E$ of what we have agreed to take as the type equivalent to $g_{1}$. Corresponding to this introduction of a parameter $\mu$ into the integrand, the function $J\left(v_{1}, \cdots, v_{n}\right)$ whose critical points correspond to the given periodic extremals will be replaced by a function $J\left(v_{1}, \cdots\right.$, 
$\left.v_{n}, \mu\right)$. In setting up $J\left(v_{1}, \cdots, v_{n}, \mu\right)$ we can and will use the same field of extremals $\left(\mathrm{IV}^{\prime}, \S 26\right)$ and corresponding parametric system $(u, v)$ as in setting up $J\left(v_{1}, \cdots, v_{n}\right)$. The broken extremals along which $J\left(v_{1}, \cdots\right.$, $\left.v_{n}, \mu_{1}\right)$ is to be the integral will, however, be taken as the extremals corresponding to the integrand in which $\mu=\mu_{1}$. Let $(A)=\left(a_{1}, \cdots, a_{n}\right)$ be the point at which the unmodified function $J\left(v_{1}, \cdots, v_{n}\right)$ has the isolated critical point corresponding to $g_{1}$. By virtue of our modification of the integrand of the problem, $J\left(v_{1}, \cdots, v_{n}, \mu_{1}\right)$ will have, in the neighborhood of the point $(A)$, a critical point $(B)$ of the type termed equivalent to that of $g_{1}$.

It follows from the lemma of this section that we can set up a function $\phi\left(v_{1}, \cdots, v_{n}\right)$ that will have in a suitably chosen neighborhood of $(A)$ no other critical point than $(B)$, and that will be identical with $J\left(v_{1}, \cdots, v_{n}\right)$ without this neighborhood of $(A)$.

We can similarly modify $J\left(v_{1}, \cdots, v_{n}\right)$ in the neighborhood of each other critical point $(A)$ that corresponds to a simply-degenerate periodic extremal $g_{1}$, so that the modified function has in the neighborhood of $(A)$ a critical point $(B)$ of a type equivalent to that of $g_{1}$, in case $g_{1}$ is convex or concave, or has no critical point at all in the neighborhood of $(A)$ in case $g_{1}$ is convexconcave, while except for the neighborhoods of these points $(A)$, the modified function is identical with the original function $J\left(v_{1}, \cdots, v_{n}\right)$.

In case the set $T$ includes doubly-degenerate periodic extremals, it follows from Theorem 7, §25, and Lemma 1 of this section, that we can first modify $J\left(v_{1}, \cdots, v_{n}\right)$ in such a fashion that the resulting function no longer has critical points corresponding to doubly-degenerate extremals. We can then, by additional successive modifications, obtain finally a function replacing $J\left(v_{1}, \cdots, v_{n}\right)$ whose critical points all correspond to non-degenerate extremals, including thereby all of the non-degenerate extremals of the original set $T$, together with the complete set of non-degenerate extremals equivalent to the degenerate extremals of $T$. The theorem then follows upon applying the lemma on critical points of $\$ 11$ to the function finally evolved from $J\left(v_{1}, \cdots, v_{n}\right)$.

\section{Part VI. Deformation theory}

28. Families of curves joining $A$ to $B$. In this part of the paper we shall show how the type of a given extremal segment can be characterized in terms of the possibility or impossibility of making certain deformations of families of curves joining $A$ to $B$. The methods will be extended to the case of closed extremals in a later section. The results obtained, it is hoped by 
the author, will serve as a part of a necessary basis for a "theory in the large" more extensive than any already developed.

By an m-family of curves, $Z_{m}$, will be understood a set of ordinary curves $(B, p .192)$ lying in the $(x, y)$ plane and passing from an initial point $A$ to $a$ final point $B$; where, further, the set of all points of $Z_{m}$ make up a single-valued continuous point function of the points on a product complex $C_{m+1}$ obtained by combining an arbitrary point $t$ on a closed interval of the $t$ axis with an arbitrary point $P$ on some $m$-dimensional manifold ${ }^{*} M_{m}$; and where, finally, the dependence of the points of $Z_{m}$ upon the points of $C_{m+1}$ is such that to hold $P$ fast and vary $t$ gives a representation of the individual curves of $Z_{m}$ by virtue 'of which they may be termed ordinary.

The point $P$ will be called the parametric point and the manifold $M_{m}$ the parametric manifold.

Let there be given two families $Z_{m}^{\prime}$ and $Z_{m}^{\prime \prime}$ consisting of curves which join the same two points $A$ and $B$. If the parametric manifolds of $Z_{m}^{\prime}$ and $Z_{m}^{\prime \prime}$ are homeomorphic the two $m$-families can be represented by the aid of the parametric manifold of either $Z_{m}^{\prime}$ or $Z_{m}^{\prime \prime}$, in particular, say, by the manifold $M_{m}$. In such a case $Z_{m}^{\prime}$ and $Z_{m}^{\prime \prime}$ will be said to be mutually deformable if corresponding to each value of a parameter $\mu$ on the interval

$$
0 \leqq \mu \leqq 1
$$

there exists an m-family $Z_{m}(\mu)$ of which $Z_{m}(0)$ is $Z_{m}^{\prime}$ and $Z_{m}(1)$ is $Z_{m}^{\prime \prime}$, while each $m$-family $Z_{m}(\mu)$ is representable in terms of the same parametric manifold $M_{m}$, and the same interval for $t$, and joins the same two points, $A$ and $B$; and if further the complete set of points $(x, y)$ on these m-families, $Z_{m}(\mu)$, by virtue of their dependence upon $\mu, t$ and $P$, make up a single-valued continuous point function of an arbitrary point on the product complex $C_{m+2}$ obtained by combining an arbitrary point $\mu$ on its interval, an arbitrary point $t$ on its interval, and an arbitrary point $P$ on $M_{m}$.

In a deformation $D$ such as the one just defined $Z_{m}^{\prime}$ will be called the initial family and $Z_{m}^{\prime \prime}$ the final family. The $m$-families $Z_{m}(\mu)$, for values of $\mu$ between 0 and 1 , will be called the intermediate families. A point $P=P_{0}$ on $M_{m}$, and a value $\mu=\mu_{0}$ held fast while $t$ varies, determine a curve in $Z_{m}\left(\mu_{0}\right)$. The curve $P=P_{0}$ of $Z_{m}^{\prime}$ will be said to be replaced in the deformation $D$ when $\mu=\mu_{0}$, by the curve $P=P_{0}$ of $Z_{m}\left(\mu_{0}\right)$. Points on a curve $P$ of $Z_{m}^{\prime}$, and points on any curve replacing $P$ under $D$ will be said to correspond if they are given by the same values of $t$. If we should hold $P$ and $t$ fast

* Cf. Veblen, The Cambridge Colloquium, 1916, Part II, Analysis Situs, p. 88. 
in $Z_{m}(\mu)$, and vary $\mu$, the resulting set of points would be the locus of points corresponding under $D$ to a single point of $Z_{m}^{\prime}$.

We hereby understand that all of the definitions of this section have been given in terms of $(u, v)$ as well as of $(x, y)$.

29. Hypotheses. Fundamental lemmas on deformations. We make here concerning $F(x, y, \dot{x}, \dot{y})$, and the given extremal $g$, the same hypothesis as in $\S 1$, except that here we suppose that $F$ and $g$ are of class $C^{\prime \prime \prime \prime}$ instead of class $C^{\prime \prime \prime}$, and that $F_{1}(x, y, \dot{x}, \dot{y})$ is positive, not only along $g$, but also for $(x, y)$ on $g$ and for $(\dot{x}, \dot{y})$ any two numbers not both zero. A consequence of the assumption that $F$ be of class $C^{\prime \prime \prime \prime}$ instead of class $C^{\prime \prime \prime}$, is that the function $J\left(v_{1}, \cdots, v_{n}\right)$ set up in $\S 1$ is here of class $C^{\prime \prime \prime}$ for $\left(v_{1}, \cdots, v_{n}\right)$ in the neighborhood of $(0, \cdots, 0)$. As in $\$ 2$ we transfer the problem to the $(u, v)$ plane, carrying $g$ into a segment $\gamma$ of the $u$ axis.

By a canonical curve will be understood a succession of extremal segments joining the successive points of the set

$$
\left(u_{0}, 0\right)\left(u_{1}, v_{1}\right), \cdots,\left(u_{n}, v_{n}\right)\left(u_{n+1}, 0\right) \text {, }
$$

determined as in $\$ 1$. An $m$-family of canonical curves will be called a canonical m-family.

Let $z$ stand for any positive constant $a, b, c, d, e$, etc. Let $R_{z}$ denote the set of points $(u, v)$ within a distance $z$ of $\gamma$.

LEMMA 1. Let $R_{1}$ be any region in the $(u, v)$ plane enclosing $\gamma$ in its interior and in which the problem is "regular." If a be a sufficiently small positive constant, the region $R_{a}$, consisting of the points $(u, v)$ within a distance $a$ of $\gamma$, will possess the following property. Any m-family $Z_{m}$ consisting of curves that lie in $R_{a}$, join $\gamma$ 's end points, and give to $J$ a value such that

$$
J \leqq J_{0}-e^{2},
$$

where $e$ is a positive constant, and $J_{0}$ is the value of $J$ along $\gamma$, can be deformed within $R_{1}$, through the mediation of curves that always satisfy (2), into an mfamily of canonical curves. This deformation can be so made that if any of the curves of $Z_{m}$ are canonical curves they are replaced in the deformation only by curves along which $v$ is a function of $u$ of at least class $C$.

Before coming to the proof proper we make a number of preliminary statements and definitions.

(A) We can and will choose a positive constant $r$ so small that of the intervals $I_{i}$ :

$$
u_{i}-r \leqq u \leqq u_{i}+r \quad(i=0,1, \cdots, n+1),
$$


no two successive intervals $I_{i}$ and $I_{i+1}$ have any points in common or contain any conjugate points of each other.

(B) If in the results obtained by Lindeberg* in a paper cited below we set

$$
G\left(x, y, x^{\prime}, y^{\prime}\right)=\left(x^{\prime 2}+y^{\prime 2}\right)^{1 / 2},
$$

we readily obtain the following. Corresponding to the positive constant $r$ just chosen in (A) there can be found a region $R_{b}$ so small that if $\bar{\gamma}$ be any "ordinary curve" (B, p. 192) joining $\gamma$ 's end points within $R_{b}$ and satisfying (2), if $u$ and $\bar{u}$ are $u$ coördinates of points $\bar{Q}$ and $Q$ on $\bar{\gamma}$ and $\gamma$ respectively, and if $\bar{Q}$ and $Q$ lie at the same distance measured along $\bar{\gamma}$ and $\gamma$ respectively from $\left(u_{0}, 0\right)$ or from $\left(u_{n+1}, 0\right)$, then

$$
|\bar{u}-u| \leqq r .
$$

(C) We will now choose a region $R_{c}$ as follows. We first require the region $R_{c}$ to lie within the region $R_{b}$ of the preceding paragraph (B). Further we can and will choose $c$ so small (B, pp. 275 and 307) that any two distinct points $Q$ and $Q^{\prime}$ which both lie in one of the regions

$$
u_{i-1}-r \leqq u \leqq u_{i}+r, \quad v \leqq c \quad(i=1,2, \cdots, n+1)
$$

can be joined in the order $Q, Q^{\prime}$ by an extremal segment $E$ with the following properties:

(a) The extremal $E$ gives a minimum to $J$ relative to all "ordinary curves" joining $Q$ to $Q^{\prime}$ and lying in (4).

(b) The coördinates $(u, v)$ of points of $E$ are functions of at least class $C^{\prime}$ of the coördinates of $Q$ and $Q^{\prime}$ and of the distance $s$ of the points $(u, v)$ from $Q$ measured along $E$.

(c) In case $Q$ and $Q^{\prime}$ lie in two successive intervals $I_{i}$ and $I_{i+1}$ of (A), the coördinate $v$ of a point $(u, v)$ on $E$ will also be a function of class $C^{\prime}$ of $u$ as well as of the coördinates of $Q$ and $Q^{\prime}$.

(D) Finally we choose a region $R_{d}$ which we will prove can serve as the region $R_{a}$ of the lemma. We first require that $d$ be so small that if the end points of any of the extremal segments $E$ described in (C) lie in $R_{d}$ the whole of $E$ will lie in $R_{c}$. A second requirement on $d$ will be added later.

Now let $Z_{m}$ be any $m$-family each curve of which lies within $R_{d}$ and satisfies the relation (2). In accordance with the conventions of $\S 28$, let $P$ be the parametric point of the family $Z_{m}$, and $M_{m}$ the parametric manifold on which $P$ lies. We come now to the deformation of $Z_{m}$ into an $m$-family of canonical curves. This deformation will be given as the resultant of two deformations,

* J. W. Lindeberg, Über einige Fragen der Variationsrechnung, Mathematische Annalen, vol. 67 (1909), p. 351, 88. 
$D^{\prime}$ and $D^{\prime \prime}$, which obviously can be combined into a single deformation if the final $m$-family of $D^{\prime}$ is identical with the initial $m$-family of $D^{\prime \prime}$.

Let each curve of $Z_{m}$ be divided into $n+1$ successive segments of which the $i$ th, say $g_{i}$, consists of points for which the arc length $s$ measured from $\left(u_{0}, 0\right)$ satisfies

$$
u_{i-1} \leqq s \leqq u_{i} \quad(i=1,2, \cdots, n),
$$

and when $i=n+1$ consists of the remaining points on the given curve. In accordance with the preceding results, (A) and (B), the coördinates of the points of $g_{i}$ will satisfy

$$
u_{i-1}-r \leqq u \leqq u_{i}+r \quad(i=1,2, \cdots, n+1) .
$$

The deformation $D^{\prime}$ will be defined as follows: For any value of the deforming parameter $\mu$ for which

$$
0<\mu \leqq 1
$$

let each arc $g_{2}$ on each curve of $Z_{m}$ be divided into two successive segments whose arc lengths are in the ratio of $\mu$ to $1-\mu$. Corresponding to the given value of $\mu$ let the second of these two segments of $g_{i}$ be replaced by itself but let the first of these two segments, say $k_{i}$, be replaced by that extremal segment, say $h_{i}$, that belongs to the class of extremals described in (C) and that joins $k_{i}$ 's end points. Points of $h_{i}$ and $k_{i}$ which divide $h_{i}$ and $k_{i}$ respectively in the same ratio as measured by arc lengths, shall be made to correspond, and shall accordingly be assigned the same values of $t$, namely the values already assigned to the points of $k_{i}$ in the representation of $Z_{m}$ in terms of $t$ and $P$.

That we have here actually defined a deformation of $Z_{m}$ readily follows from (C). Let $Z_{m}{ }^{\prime}$ denote the final $m$-family of the deformation $D^{\prime}$. According to paragraph (D) the curves of $Z_{m}^{\prime}$ will all lie in $R_{c}$, and according to paragraph (C), (c), their $v$ coördinates will be functions of their $u$ coördinates of class $C$ at least. Further, the curves used in the deformation $D^{\prime}$ give to $J$ at most the value which the corresponding curves of $Z_{m}$ give, so that the curves used in $D^{\prime}$ satisfy ( 2 ).

We can now define a second deformation $D^{\prime \prime}$ under which $Z_{m}^{\prime}$ will be deformed into a family of canonical curves satisfying (2). The definition of $D^{\prime \prime}$ is similar to that of $D^{\prime}$ except that the segments $g_{i}$ into which we divide the curves of $Z_{m}^{\prime}$ can here be defined in terms of $u$ instead of $s$ as follows:

$$
u_{i-1} \leqq u \leqq u_{i} \quad(i=1,2, \cdots, n+1) .
$$

The combination of the deformations $D^{\prime}$ and $D^{\prime \prime}$ will obviously give a deformation of the desired sort and the lemma is proved except for its 
concluding sentence. This concluding sentence is also readily seen to be true if the constant $d$ of $(D)$ be further restricted in magnitude so that the slopes of the canonical curves in $Z_{m}$ will be less in absolute value than a sufficiently small positive constant.

In the deformation $D^{\prime \prime}$ a curve which initially was canonical is for each value of the deforming parameter $\mu$ replaced by itself. If in the original $m$-family the variable $t$ had been $u$, then of the deformations $D^{\prime}$ and $D^{\prime \prime}$ only $D^{\prime \prime}$ would have been needed. We thus have the following lemma.

Lemma 2. If in Lemma 1 an m-family $Z_{m}$ is given in which the variable $t=u$, then the deformation whose existence is affirmed in Lemma 1 can be defined in such a fashion that any curve of $Z_{m}$ which is a canonical curve remains unaltered throughout the deformation.

The following Lemma is important.

LeMma 3. Let $R_{1}$ be a region of the $(u, v)$ plane enclosing $\gamma$ in its interior, and in which the problem is regular. If $e^{\prime}$ be any sufficiently small positive constant, the region $R_{\boldsymbol{o}^{\prime}}$ consisting of points $(u, v)$ within a distance $e^{\prime}$ of $\boldsymbol{\gamma}$ has the following property. If a canonical m-family $Z_{m}^{\prime}$ can be deformed within $R_{e^{\prime}}$ into a canonical m-family $Z_{m}^{\prime \prime}$ through a deformation $D$ all of whose curves give to $J$ a value such that

$$
J \leqq J_{0}-e^{2},
$$

where $e$ is a positive constant, and $J_{0}$ the value of $J$ along $\gamma$, then $Z_{m}^{\prime}$ can be deformed into $Z_{m}^{\prime \prime}$, within $R_{1}$, through the mediation of curves all of which are canonical and which satisfy (6).

For a moment suppose the region $R_{1}$ given in Lemma 1 is the region $R_{1}$ given in the present lemma. Corresponding to this choice of $R_{1}$ let $R_{a^{\prime}}$ be a particular choice of the region $R_{a}$ such that Lemmas 1 and 2 hold true. Now let us apply Lemma 1 again, this time taking for the region $R_{1}$ of Lemma 1 the region $R_{a^{\prime}}$ just determined. Corresponding to this second choice of $R_{1}$, Lemma 1 will hold true for a second proper choice of $R_{a}$ which we now denote by $R_{a^{\prime \prime}}$. We will prove Lemma 3 is true if $R_{e^{\prime}}$ in Lemma 3 be taken as $R_{a^{\prime \prime}}$.

We first note that the complete set of curves used in the deformation $D$ can be considered as an $(m+1)$-family $Z_{m+1}$. For if $M_{m}$ is the common parametric manifold of $Z_{m}^{\prime}$ and $Z_{m}{ }^{\prime \prime}$, each curve of $D$ is specified by a pair $(P, \mu)$, where $P$ is on $M_{m}$ and $\mu$ on its interval $(0,1)$. These curves of $D$ can then be regarded as specified by a point $P_{1}=(P, \mu)$ the totality of which points form an $(m+1)$-dimensional manifold $M_{m+1}$. 
Now the ( $m+1)$-family $Z_{m+1}$ can be deformed subject to (6), and within $R_{a^{\prime}}$, into a canonical $(m+1)$-family $\bar{Z}_{m+1}$ using thereby the deformation, say $\bar{D}$, of Lemma 1 . Under $\bar{D}$ the given canonical $m$-families $Z_{m}^{\prime}$ and $Z_{m}^{\prime \prime}$ appear initially in $Z_{m+1}$, and will be replaced finally in $\bar{Z}_{m+1}$ by canonical families, say $\bar{Z}_{m}^{\prime}$ and $\bar{Z}_{m}{ }^{\prime \prime}$ respectively. We will prove the lemma by showing how each of the first three canonical $m$-families of the set

$$
Z_{m}{ }^{\prime}, \bar{Z}_{m}{ }^{\prime}, \bar{Z}_{m}{ }^{\prime \prime}, Z_{m}{ }^{\prime \prime}
$$

can be deformed, subject to (6), into its successor in (7) by a deformation in which only canonical curves are used.

(a) The deformation of $Z_{m}^{\prime}$ into $\bar{Z}_{m}{ }^{\prime}$. We may fix our attention upon those curves of $\bar{D}$ which serve to deform $Z_{m}^{\prime}$ into $\bar{Z}_{m}^{\prime}$ and let $Z_{m+1}^{\prime}$ be the $(m+1)$-family of curves in $\bar{D}$ replacing $Z_{m}^{\prime}$, including also $Z_{m}^{\prime}$ and $\bar{Z}_{m}^{\prime}$.

According to the concluding sentence of Lemma $1, Z_{m+1}^{\prime}$ consists of curves along which $v$ is a function of $u$ of class $C$. According to Lemma 2, $Z_{m+1}$ can then be carried by a deformation $D^{\prime}$ which does not alter $Z_{m}^{\prime}$ and $\bar{Z}_{m}{ }^{\prime}$ into a set of canonical curves. The final canonical $(m+1)$-family of curves of $D^{\prime}$ may be considered as the curves of the desired deformation, say $D_{1}$, of $Z_{m}^{\prime}$ into $\bar{Z}_{m}{ }^{\prime}$.

(b) The deformation of $\bar{Z}_{m}^{\prime}$ into $\bar{Z}_{m}^{\prime \prime}$. The deformation, say $D_{2}$, required here is furnished by the set of canonical curves which make up $\bar{Z}_{m+1}$.

(c) The deformation of $\bar{Z}_{m}^{\prime \prime}$ into $Z_{m}^{\prime \prime}$. This deformation, say $D_{3}$, is set up as $D_{1}$ was set up in (a).

The combination of $D_{1}, D_{2}$, and $D_{3}$, in the order written, will give the required deformation of $Z_{m}^{\prime}$ into $Z_{m}^{\prime \prime}$ and the lemma is proved.

30. The analysis situs of the deformation problem. We have been denoting by $J\left(v_{1}, \cdots, v_{n}\right)$ the value of the integral $J$ along the canonical curves joining the points $(1)$ of $\$ 29$. If $\left(u_{0}, 0\right)$ is not conjugate to $\left(u_{n+1}, 0\right)$ but if there are $k$ conjugate points of $\left(u_{0}, 0\right)$ preceding $\left(u_{n+1}, 0\right)$, it follows from Theorem 2 of $\S 6$ that $J\left(v_{1}, \cdots, v_{n}\right)$ has a critical point of rank $n$ and type $k$ at the point $\left(v_{1}, \cdots, v_{n}\right)=(0, \cdots, 0)$. It follows from a lemma proved by the author* that there exists a one-to-one continuous transformation of the variables $\left(v_{1}, \cdots, v_{n}\right)$ into variables $\left(y_{1}, \ldots, y_{n}\right)$ in which the point $\left(v_{1}, \cdots, v_{n}\right)=(0, \cdots, 0)$ corresponds to $\left(y_{1}, \cdots, y_{n}\right)=(0, \cdots, 0)$, and under which

$$
\begin{aligned}
J\left(v_{1}, \cdots, v_{n}\right)-J(0, \cdots, 0) & \\
& =-y_{1}^{2}-y_{2}^{2}-\cdots-y_{k}^{2}+y_{k+1}^{2}+\cdots+y_{n}^{2}
\end{aligned}
$$

* Marston Morse, these Transactions, loc. cit., p. 354. 
for $\left(y_{1}, \cdots, y_{n}\right)$ in the neighborhood of $(0, \cdots, 0)$. We exclude for the present the case where $k=0$.

For the sake of brevity we set

$$
\begin{array}{ll}
p^{2}=y_{1}^{2}+y_{2}^{2}+\cdots+y_{k}^{2}, & 0<k \leqq n, \\
q^{2}=y_{k+1}^{2}+y_{k+2}^{2}+\cdots+y_{n}^{2}, &
\end{array}
$$

understanding $q^{2}$ to be identically zero if $k=n$. Now let $a$ be a positive constant so small that the domain

$$
p^{2}+q^{2} \leqq a^{2}
$$

is one for which (1) holds as described.

In the present section we shall concern ourselves with deformations, in the neighborhood of the extremal segment $\gamma$, of $m$-families $Z_{m}$ composed of canonical curves subject to a condition of the form

$$
J\left(v_{1}, \cdots, v_{n}\right) \leqq J(0, \cdots, 0)-e^{2}, \quad e<a,
$$

where $e$ is an arbitrarily small positive constant. We shall confine ourselves to $m$-families corresponding to which the parametric manifold $M_{m}$ is closed. Corresponding to each point $P$ on $M_{m}$ there is a curve on the given $m$-family $Z_{m}$. In the present case each such curve is canonical, and the points at which it crosses the straight lines $u=u_{i}$ have for their $v$ coördinates $v_{i}$. Thus each point $P$ on $M_{m}$ determines a point $\left(v_{1}, \cdots, v_{n}\right)=(V)$. If we regard the points $(V)$ as distinct when they arise from distinct points $P$ on $M_{m}$, the set of all such points $(V)$ form a closed $m$-dimensional manifold $M_{m}^{\prime}$ homeomorphic with the manifold $M_{m}$. If the given canonical $m$-family be deformed in the sense of $\$ 28$, the manifold $M_{m}^{\prime}$ arising at each stage of the deformation from the $m$-family which replaces $Z_{m}$ at that stage, will itself be "homotopically deformed" in the sense of analysis situs.*

We turn next to the space of the points $(Y)=\left(y_{1}, \cdots, y_{n}\right)$ and confine ourselves to a spherical neighborhood of the origin in the form (4), and to points in the space of the points $(V)$ that correspond to the neighborhood in the space of the points $(Y)$. Corresponding to a deformation of $M_{m}^{\prime}$ in the space of the points $(V)$ we shall have to deal with a deformation of a corresponding manifold $M_{m}^{\prime \prime}$ in the space of the points $(Y)$. Our requirement that the curves of the given $m$-family satisfy (5) becomes in terms of the points $(Y)$ on the manifold $M_{m}^{\prime \prime}$ the condition

$$
p^{2}-q^{2} \geqq e^{2}, \quad 0<k \leqq n .
$$

* Veblen, loc. cit., pp. 125-126. 
31. Homotopic deformations in the space $(Y)$. The preceding condition (6) and the restriction of the points $(Y)$ to points in a spherical neighborhood of $(Y)=(0, \cdots, 0)$ combine to give us the relations

$$
\begin{array}{ll}
p^{2}+q^{2} \leqq a^{2}, & 0<e<a, \\
p^{2}-q^{2} \geqq e^{2}, & 0<k \leqq n .
\end{array}
$$

The relations (1) define a finite closed region in the space of the points $(Y)$. The deformation of $m$-families thus leads us to study deformations of closed manifolds in the space (1).

We could get some but not all of the properties of the space (1) by breaking it up into cells, and showing that of its connectivity numbers

$$
R_{0}, R_{1}, \cdots, R_{n}
$$

all are unity except one, namely

$$
R_{k-1}=2 \text {. }
$$

Note that the $(k-1)$-dimensional sphere

$$
p^{2}=e^{2}, \quad q^{2}=0
$$

is among the points of (1). Denote this sphere by $S_{k-1}$. A very important fact is that the region (1) can be deformed through the mediation of its own points into a singular complex on $S_{k-1}$. In terms of a deforming parameter $\boldsymbol{\mu}$ the set of intermediate and final positions, $\left(y_{1}, \cdots, y_{n}\right)$, corresponding to any initial point $\left(a_{1}, \cdots, a_{n}\right)$ of $(1)$, in a deformation $D$ of (1) into a complex on $S_{k-1}$ can be given as follows:

where

$$
\begin{array}{r}
y_{i}^{2}=(1-\mu) a_{i}^{2}+\frac{\mu e^{2} a_{i}^{2}}{a_{1}^{2}+\cdots+a_{k}^{2}} \quad(i=1,2, \cdots, k), \\
y_{i}^{2}=(1-\mu) a_{i}^{2} \quad(i=k+1, k+2, \cdots, n),
\end{array}
$$

$$
0<\mu \leqq 1,
$$

and where the coördinates $\left(y_{1}, \cdots, y_{n}\right)$ are respectively required to have the sign of the coördinates $\left(a_{1}, \cdots, a_{n}\right)$ from which they are deformed.

We can now prove that any $m$-dimensional closed manifold $M_{m}$, singular or non-singular, lying on (1) and such that $m$ is less than $k-1$, can be deformed on (1) into a point. For under the above deformation (3), $M_{m}$ can be deformed into a manifold $M_{m}^{\prime}$ on $S_{k-1}$. Regardless of whether $M_{m}^{\prime}$ is singular or non-singular on $S_{k-1}$, there is a fundamental theorem on homotopic deformations* to the effect that $M_{m}^{\prime}$ can be deformed on $S_{k-1}$ into a manifold

- Veblen, loc. cit., p. 131. 
$M_{m}^{\prime \prime}$ on $S_{k-1}$ each of whose cells "covers" a cell of $S_{k-1}$. Hence $M_{m}^{\prime \prime}$ can be deformed into a point on $S_{k-1}$; and the statement in italics follows at once.

The sphere $S_{k-1}$ cannot itself be deformed on (1) into a point on (1). For if there were such a deformation, say $D^{\prime}$, let the family of intermediate manifolds homeomorphic with $S_{k-1}$ under $D^{\prime}$ be deformed onto $S_{k-1}$ under the deformation (3). The resulting family of manifolds on $S_{k-1}$ would constitute a deformation on $S_{k-1}$ in which the initial manifold would cover $S_{k-1}$ just once and the final manifold coincide with a point. This, however, is impossible according to a fundamental theorem of analysis situs.*

We can now add the statement that $S_{k-1}$ cannot be deformed on (1) into any manifold on a manifold $M_{r}$ on (1) of lower dimension than $k-1$. For according to the preceding paragraph, $M_{r}$, and hence $S_{k-1}$, could be deformed on (1) into a point on (1). By making use of product manifolds analogous to surfaces of revolution in 3-space it is easy to set up for each $m>k-1$ an example of a manifold on (1) which cannot be deformed on (1) into any manifold of dimensionality less than $k-1$.

Finally with the aid of the deformation (3) we obtain the following result. Any closed manifold $M_{m}$ on (1) for which $m \geqq k-1$ can always be deformed on (1) into a manifold on $S_{k-1}$, and in special cases can be further deformed on $S_{k-1}$ into a point.

32. The theorem on deformations of $m$-families joining $A$ to $B$. We can now translate the results of the preceding section into terms of deformations of canonical $m$-families. We shall understand that an $m$-family shall be considered as "on" an r-family if every curve of the m-family coincides with some curve of the r-family. Let it also be understood that a closed $m$ family shall mean an m-family whose parametric manifold is closed. In particular it should be noted that a closed 0-family means a pair of ordinary curves joining the same two points. The results of the preceding section now give the following lemma.

LEMma. Let $\gamma$ be the extremal segment $v=0$ of $\$ 29$. Suppose there are $k$ points $(k>0)$, conjugate to $\gamma$ 's initial point $A$ and preceding $\gamma$ 's final point $B$, but that $A$ is not conjugate to $B$. Then within any region $R$ enclosing $\gamma$ in its interior there exists a region $R_{1}$ enclosing $\gamma$ in its interior, and corresponding to $R_{1}$ an arbitrarily small positive constant such that the closed $m$-families of canonical curves that satisfy

$$
J \leqq J_{0}-e^{2}
$$

and lie in $R_{1}$ are conditioned as follows:

* Veblen, p. 131, §14. 
(a) Those for which $m<k-1$ can be deformed among canonical curves, within $R_{1}$, and subject to (1), into a single canonical curve.

(b) Those for which $m \geqq k-1$ include for each $m$ at least one $m$-family that cannot be deformed among canonical curves, within $R_{1}$, and subject to (1), into a single canonical curve, or even into a $(k-1)$-family on an $r$-family for which $r<k-1$.

(c) Those for which $m \geqq k-1$ can always be deformed among canonical curves, within $R_{1}$, and subject to (1), into an m-family of canonical curves on a $(k-1)$-family $Z_{k-1}$, and, in special cases, can be further deformed into a single canonical curve.

This lemma follows from the italicized statements of the preceding section, taking for $Z_{k-1}$ the $m$-family determined by the points $(V)$ that correspond to the points $(Y)$ on $S_{k-1}$.

Lemmas $1,2,3$ of $\$ 29$ tell how and when any $m$-family of ordinary curves in the $(u, v)$ plane can be deformed into a closed $m$-family of canonical curves. The preceding lemma describes the limitations on the deformations of closed $m$-families of canonical curves. The combination of the results of these lemmas carried over into the $(x, y)$ plane gives the following fundamental theorem. (Here it will be convenient to call a region enclosing $g$ in its interior, and consisting of points $(x, y)$ within an arbitrarily small positive constant distance of $g$, an arbitrarily small neighborhood of $g$. The term a sufficiently small neighborhood of $g$ will be similarly defined.)

Theorem 9. Let there be given in the $(x, y)$ plane the extremal $g$ of $\$ 29$.

On $g$ let there be $k$ points conjugate to $A, k>0$, but suppose $B$ is not conjugate to $A$. Let $J_{0}$ be the value of the integral $J$ along $g$.

Then corresponding to any sufficiently small neighborhood $R$ of $g$, there exists within $R$ an arbitrarily small neighborhood $R_{1}$ of $g$, and an arbitrarily small positive constant $e$ with the following properties. Closed $m$-families of curves which lie in $R_{1}$, which join $g$ 's end points, and give to the integral $J$ a value for which (1) holds, are conditioned as follows:

(a) Those for which $m<k-1$ can be deformed in $R$ and subject to (1) into a single curve.

(b) Those for which $m \geqq k-1$ include for each $m$ at least one $m$-family that cannot be deformed, within $R$ and subject to (1), into any $(k-1)$-family on an r-family for which $r<k-1$, or into a single curve.

(c) Those for which $m \geqq k-1$ can always be deformed, within $R$ and subject to (1), into an m-family on a $(k-1)$-family, and in special cases can be further deformed into a single curve. 
There remains the case where $A$ is conjugate to $B$. Here we need to assume that the problem is analytic, as well as regular, in the neighborhood of $g$. We suppose that not every extremal through $A$ with slope at $A$ near that of $g$ passes through $B$. In accordance with the conventions preceding Theorem $3, \S 12, g$ will have a type number $k=s$, or $k=s+1$, or type numbers $k=s$ and $s+1$, where $s$ is the number of points on $g$ conjugate to $A$ and preceding $B$. It can be shown that if $k=s$, or $k=s+1$, the preceding theorem holds if the sentence concerning conjugate points be omitted, while if $g$ is of the composite type where $k=s$ and $k=s+1$, the preceding theorem should be further altered by replacing (a), (b), and (c) by the statement that any mfamily whatsoever lying in $R_{1}$, joining $A$ to $B$, and satisfying (1) can be deformed within $R$ and subject to (1), into a single curve joining $A$ to $B$.

A proof of these last facts would lead us too far astray. The writer hopes to return to a discussion of critical points of rank less than $n$ in a separate paper.

33. An illustrative example. Consider the equator of a unit sphere. Refer the sphere near $C$ to coördinates $(u, v)$, giving respectively the latitude and longitude of the point. Consider the sphere near $C$ as now covered an infinite number of times by an unending strip $S$ which overhangs the neighborhood of $C$ after the manner of a Riemann surface. We may suppose $S$ represented in the $(u, v)$ plane by an unending strip $R$ containing the $u$ axis. We suppose our integral corresponds to the arc length on $S$.

The case $k=1$. If we take a segment of the $u$ axis whose length $g$ lies between $\pi$ and $2 \pi$ we will have the case $k=1$ of the preceding theorem. In the strip $R$ we can clearly take two curves which join $g$ 's end points but otherwise lie on opposite sides of $g$, and whose lengths are equal but both less than $g$. These two curves form a 0 -family which cannot be deformed into a single curve without passing out of $R$ or increasing the lengths on $S$.

The case $k=2$. Suppose now that the length $g$ of $g$ is between $2 \pi$ and $3 \pi$. Here we have the case $k=2$. Let $u_{0}$ and $u_{3}$ correspond to the end points of $g$, and let $u_{0}, u_{1}, u_{2}, u_{3}$ correspond to four successive points on the $u$ axis such that no one of the resulting three successive intervals is as great as $\pi$ in length. We consider a one-dimensional closed manifold of pairs $\left(v_{1}, v_{2}\right)$, namely the pairs

$$
v_{1}(a)=b \cos a, \quad v_{2}(a)=b \sin a,
$$

where $a$ is any real number, and is to be our parameter, and $b$ is a positive constant. If $b$ be sufficiently small, the following will hold true. On $S$ the images of the points

$$
\left[u_{0}, 0\right],\left[u_{1}, v_{1}(a)\right],\left[u_{2}, v_{2}(a)\right],\left[\left(u_{3}, 0\right]\right.
$$


can be successively joined by arcs of great circles which will give the shortest paths between their end points. The images on $R$ of all such paths for all values of $a$ will give a closed 1 -family. The lengths of the different paths of the family considered as a function of $a$ will have a maximum $M$ which is readily seen to be less than $g$. If our choice of $b$ be sufficiently small, it follows from the general theory that this 1-family cannot be deformed into a single curve joining $g$ 's end points without increasing the lengths beyond $M$ or passing out of $R$.

If however we join the end points of $g$ by any two ordinary curves whose lengths on $S$ are less than a constant $g-e<g$, and which lie in a sufficiently small neighborhood of $g$, then, according to the preceding theorem, these two curves can be deformed into each other without passing out of $R$, or using curves whose lengths exceed $g-e$.

34. Osgood's Theorem. It has doubtless been noted that the case where $k=0$ has been omitted in the preceding discussion for the reason that there are in this case no curves neighboring the given extremal segment which satisfy the inequality (1) of the preceding section. For the case $k=0$ it seems to the author that the theorem that is due to Osgood* represents the nearest approach to the preceding developments. The questions at issue are sufficiently similar to have suggested to the author $a$ proof of Osgood's Theorem in the form stated by Hahn $(B, p .281)$ without, however, the hypothesis, used by Hahn and by recent writers on the calculus of variations, that at the ends of the extremal segment the problem be regular. Such a proof seems desirable because it reduces the hypotheses under which Osgood's Theorem can be proved to a set identical with the least hypotheses under which the proper strong minimum is ordinarily established. This proof will be published later.

35. Deformations of periodic extremals. Although the results here are different from those of the preceding sections yet the methods may be carried over, and afford a proof of the desired results once the problem is well defined.

By an $m$-family of closed curves $Z_{m}$ will be understood a set of ordinary closed curves in the $(x, y)$ plane, the set of all of whose points make up a single-valued, continuous, point function of the point on a product complex $C_{m+1}$, obtained by combining an arbitrary point $Q$ on a unit circle with an arbitrary point $P$ on some $m$-dimensional manifold $M_{m}$. The dependence of the points of $Z_{m}$ upon the points of $C_{m+1}$ is to be such that each individual closed curve is obtained by holding $P$ fast and varying $Q$ on the unit circle;

* Osgood, these Transactions, vol. 2 (1901), p. 273. 
and the dependence of the variable point of each individual curve upon $Q$, or more particularly upon the arc length measured from a fixed point on the unit circle to the point $Q$, is to be one by virtue of which the curve may be termed ordinary.

The remaining definitions and results of $\S \S 28,29,30,31$, and 32 , may be carried over here either unchanged or with the changes obvious at each point. We have the following central theorem:

THEOREM 10. Let there be given an integrand $F(x, y, \dot{x}, \dot{y})$ satisfying the hypotheses of $\$ 1$ except that here $F$ is to be of class $C^{\prime \prime \prime \prime}$. Let there be given in $S$ a non-degenerate ( $\$ 17)$ periodic extremal $g$, at every point $(x, y)$ of which, and for every $\theta, F_{1}(x, y, \cos \theta, \sin \theta)$ is positive. Let $k$ be the type number of $g$, determined as in Theorem 5, §21. Let $J_{0}$ be the value of $J$ along $g$.

Then corresponding to any sufficiently small neighborhood $R$ of $g$, there exists within $R$ an arbitrarily small neighborhood $R_{1}$ of $g$, and an arbitrarily small positive constant $e$ with the following properties. Closed $m$-families of periodic curves which are deformable within $R_{1}$ into $g$, and which give to the integral $J$ a value such that $J<J_{0}-e^{2}$ satisfy the statements (a), (b), and (c) of Theorem 9.

36. Birkhoff's Theorem.* In dealing with deformations of one closed trajectory into another Birkhoff has given a theorem which for the case of dynamical systems is essentially equivalent to that special part of the preceding theorem which has to do with the mutual deformability of two closed trajectories. Birkhoff does not consider such entities as $m$-families in general. However his pair of closed trajectories comes under the head of what the author has called closed 0-families. Birkhoff's theorem is stated in terms of the Poincare rotation number, and in terms of that rotation number tells when a pair of closed trajectories can be deformed into each other. The author's theorem shows that periodic extremals for which $k>1$ cannot be distinguished in type simply by a consideration of the mutual deformability of two closed trajectories. It should be stated in explanation that Birkhoff was not seeking to distinguish between all types of periodic extremals by means of deformations, but rather to determine to what types his "minimax principle" applied, and for that purpose his theorem was sufficient.

Speaking generally, the results of Part VI show that the number of conjugate points on extremals joining two fixed points, or the type number of periodic extremals, is the least integer $m$ for which some $(m-1)$-family of curves

* Birkhoff, loc. cit., p. 249. 
cannot be deformed into a single curve subject to the conditions described in the text. This result suggests another new and powerful way of approaching a theory "in the large."

\section{PART VII. VARIABLE END POINTS}

37. A geometric theorem. No general development for this case will be presented. However, a suggestive and intrinsically complete geometric theorem has been obtained in a problem that comes under this head, namely the problem of the number and nature of the normals which can be drawn from a fixed point $P$ in a euclidean $(n+1)$-space $E_{n+1}$ to an $n$-spread $S_{n}$ in that space. Proofs of the following results will be published later.

The $n$-spread $S_{n}$ is to be a manifold without boundary in the sense of Veblen (loc. cit.). Moreover we assume that the points on $S_{n}$ in the neighborhood of any given point of $S_{n}$ are representable by an equation which gives one of the rectangular coördinates as a function of class $C^{\prime \prime}$ of the others. A preliminary result is that the loci of centers of principal normal curvature* $C_{n}$ corresponding to $S_{n}$ are not space filling in $E_{n+1}$, that is, there are in the neighborhood of each point of $E_{n+1}$ points not on $C_{n}$. Let $P$ be any point not on $C_{n}$ or $S_{n}$. A second preliminary result is that any straight line $L$ through $P$ will be normal to $S_{n}$ at no more than a finite number of points $Q_{i}(i=1,2$, . . , m). Each of the $m$ segments $P Q_{i}$ of $L$ will be counted as a different normal from $P$ to $S_{n}$. A normal $P Q_{i}$ will be said to be of type $k$ if there are $k$ centers of principal normal curvature corresponding to $Q_{i}$, on $L$, between $P$ and $Q_{i}$. We have the following theorem.

TheOREM. Let $P$ be any point not on the manifold $S_{n}$, nor on the loci of centers of principal normal curvature of $S_{n}$, and let $M_{k}$ be the number of different normals from $P$ to $S_{n}$ of type $k$. Then between the numbers $M_{k}$, and the connectivities $R_{i}$ of $S_{n}$, the following relations hold true:

$$
\begin{aligned}
& M_{0} \geqq 1+\left(R_{0}-1\right), \\
& M_{0}-M_{1} \leqq 1+\left(R_{0}-1\right)-\left(R_{1}-1\right) \text {, } \\
& M_{0}-M_{1}+M_{2} \geqq 1+\left(R_{0}-1\right)-\left(R_{1}-1\right)+\left(R_{2}-1\right) \text {, } \\
& M_{0}-M_{1}+\cdots+(-1)^{n} M_{n}=1+\left(R_{0}-1\right)-+\cdots+(-1)^{n}\left(R_{n}-1\right) \text {, }
\end{aligned}
$$

where the inequality signs alternate until the final equality is reached.

\footnotetext{
* Eisenhart, Riemannian Geometry, p. 153.
} 
To this theorem* we add the result that points $Q$ on $S_{n}$ such that the straight line segment $P Q$ gives a relative minimum or maximum to the distance from $P$ to $S_{n}$, yield normals $P Q$, respectively of types 0 or $n$. We now make the following specific applications of the theorem.

In 3-space let $S_{2}$ be homeomorphic to a sphere. Here

$$
R_{0}=1, R_{1}=1, R_{2}=2 \text {. }
$$

If there are $r$ normals from $P$ to $S_{2}$ which give a relative minimum to the distance from $P$ to $S_{2}$, and $s$ normals that give a relative maximum, there are $r+s-2$ other normals of type 1 . The total number of normals is always even.

In 3-space let $S_{2}$ be homeomorphic to a torus. Here

$$
\begin{aligned}
& R_{0}=1, \quad R_{1}=3, \quad R_{2}=2, \\
& M_{0} \geqq 1, \quad M_{1} \geqq 2, \quad M_{2} \geqq 1 .
\end{aligned}
$$

Thus there are always four normals at least. The total number of normals is always even.

In 4-space let $S_{3}$ be homeomorphic to a manifold obtained by identifying the opposite faces of an ordinary cube. Here

$$
\begin{aligned}
& R_{0}=1, \quad R_{1}=4, \quad R_{2}=4, \quad R_{3}=2, \\
& M_{0} \geqq 1, \quad M_{1} \geqq 3, \quad M_{2} \geqq 3, \quad M_{3} \geqq 1 .
\end{aligned}
$$

There are thus at least eight normals, while the total number of normals is always even.

* A theorem essentially the same as the above has been proved to hold in the general problem one variable end point in $m$ dimensions.

harvard Universtty,

Cambridge, Mass. 